\title{
The wisdom of the crowd in dynamic economies
}

\author{
Pietro Dindo \\ Department of Economics, Ca' Foscari University of Venice
}

Filippo MAssari

Department of Decision Sciences, Bocconi University and School of Economics, University of East Anglia

The wisdom of the crowd applied to financial markets asserts that prices represent a consensus belief that is more accurate than individual beliefs. However, a market selection argument implies that prices eventually reflect only the beliefs of the most accurate agent. In this paper, we show how to reconcile these alternative points of view. In markets in which agents naively learn from equilibrium prices, a dynamic wisdom of the crowd holds. Market participation increases agents' accuracy, and equilibrium prices are more accurate than the most accurate agent.

KeYwords. Wisdom of the crowd, heterogeneous beliefs, market selection hypothesis, naive learning.

JEL ClASSificATION. D01, D53, G1.

\section{INTRODUCTION}

The informational content of prices is a central issue in the analysis of equilibria of competitive markets. In financial markets, in particular, asset prices are often believed to be good predictors of the economic performance of the underlying fundamentals. Three different mechanisms have been proposed as possible explanations for this remarkable property. The rational expectation and the learning from price literatures argue that equilibrium prices are accurate because they reveal and aggregate the information of all market participants. The Market Selection Hypothesis, MSH, proposes instead that prices become accurate because they eventually reflect only the beliefs of the most accurate agent. The Wisdom of the Crowd, WOC, suggests that market prices are accurate because individual, opposite biases are averaged out by the price formation mechanism.

Although these theories aim to explain the same phenomenon, they rest on different and somehow conflicting hypotheses. In the learning from price literature, all agents are

Pietro Dindo: pietro.dindo@unive.it

Filippo Massari: massari3141@gmail.com

We wish to thank Larry Blume, Giulio Bottazzi, David Easley, Daniele Giachini, Itzhak Gilboa, Ani Guerdjikova, Willemien Kets, Alvaro Sandroni, Rajiv Sethi, Jan Werner, as well as conference and seminar participants at ESEM 2017 (Lisbon), SAET 2017 (Faro), Bocconi University, Catholic University (Milan), the Cowles Foundation's 13th Annual Conference on General Equilibrium and its Applications, RoDeO (Venice), ASSET 2016 (Thessaloniki), and IMPA (Rio de Janeiro) for their comments and suggestions. Pietro Dindo acknowledges the support of the Marie Curie International Outgoing Fellowship PIOF-GA-2011-300637 within the 7th European Community Framework Programme and the hospitality of the Department of Economics at Cornell University.

(C) 2020 The Authors. Licensed under the Creative Commons Attribution-NonCommercial License 4.0. Available at https://econtheory.org. https://doi.org/10.3982/TE3924 
assumed to agree on the way to interpret information. In equilibrium, when all private information gets revealed, all agents must hold the same belief because they cannot "agree to disagree." Therefore, the MSH and the WOC arguments are void. By contrast, in the MSH and the WOC literatures, agents can disagree on how to interpret information about fundamentals. However, existing models of market selection are incompatible with the WOC because they do not allow for belief heterogeneity in the long run: by selecting the most accurate agent, the market destroys all accuracy gains that could be achieved by balancing out agents' opposite biases. Focusing on static settings, the WOC literature struggles to justify the assumption that the joint distribution of consumption shares and beliefs is such that the opposite biases of agents cancel out.

In this paper, we provide conditions for the WOC to occur in dynamic economies. We extend the general equilibrium model of market selection of Sandroni (2000) and Blume and Easley (2006) by allowing the beliefs of some agents to depend on an endogenous market consensus. The one-period-ahead beliefs of these agents are formed by giving weight to two different models. The first model, market consensus, is generated endogenously by the market and shared by all agents. The second model, dogmatic probabilities, is agent-specific and represents their subjective probabilistic view.

We provide sufficient conditions on agents' dogmatic probabilities, model weights, and preferences such that the WOC occurs in equilibrium: irrespective of the initial consumption share distribution, selection forces endogenously determine a consumption share dynamics that makes the market consensus more accurate than the most accurate agent in isolation. Furthermore, we show that if some agents have dogmatic beliefs with opposite bias, the consensus becomes as accurate as the truth in the limit of these agents relying only on the consensus.

The intuition for the occurrence of the WOC is as follows. Imagine two agents, 1 and 2, whose dogmatic probabilities have an opposite bias, e.g., agent 1 being too optimistic about a state of the world, while the other is too pessimistic. Agents are allowed to trade on these differences of opinion and, in equilibrium, the optimist gains wealth when such a state is realized. If, due to a lucky initial draw, agent 1 accumulates a substantial wealth share, then the market consensus will converge to his belief. Agent 2's belief, being the weighted average of his pessimistic belief and the market consensus (which is now optimistic), becomes closer to the truth. Hence, agent 2 starts accumulating wealth, on average, and the market consensus shifts toward his dogmatic belief. However, agent 2 cannot accumulate wealth for too long, otherwise he makes agent 1 the most accurate. So, heterogeneity is persistent and the WOC emerges because the market consensus is on average closer to the truth than either of the two dogmatic beliefs.

The dynamics of our economy depends crucially on the definition of the consensus. We propose two measures of consensus, the market probabilities and the riskneutral probabilities. The rationale behind both measures is that they weight the beliefs of agents with larger consumption shares more than those of agents with smaller consumption shares so that the consensus obtained in an economy with a unique agent coincides with his beliefs. The market probabilities make the dynamics of beliefs and the occurrence of the WOC qualitatively independent of risk attitudes and the aggregate endowment process, but require detailed information about agents' beliefs, preferences, 
and consumption shares to be computed. The risk-neutral probabilities can be calculated from state prices and knowledge of the aggregate endowment process alone, but make the dynamics of beliefs and the occurrence of the WOC dependent on risk attitudes.

The following describes the structure of the paper and our main findings. In Section 2, we introduce the model of the economy, agents' beliefs, the market consensuses, belief and market accuracy, and define the WOC as the situation in which the consensus is more accurate than all dogmatic probabilities.

In Section 3, we focus on the case in which the consensus is the market probability. We show that the WOC emerges when at least two agents with opposite bias sufficiently weigh the consensus in forming their beliefs. In this case, the equilibrium path exhibits long-run heterogeneity, market probabilities never settle down, and selection forces generate endogenously a dynamics of the joint distribution of consumption shares and beliefs that determines the WOC. Moreover, we demonstrate that market accuracy is a virtuous self-fulfilling prophecy. If some agents with opposite bias are almost certain that the consensus is correct, the consensus is indeed almost correct. In the limit, selection forces endogenously determine a consumption share dynamics such that, in equilibrium, the consensus coincides with the true probability.

Last, in Section 4, we extend our analysis to the case in which agents use the riskneutral probability for consensus and characterize how risk attitudes affect the riskneutral consensus accuracy and the beliefs dynamics. We consider economies in which all agents have CRRA utility and show that, ceteris paribus, economies with more riskaverse agents generate a more accurate market consensus than economies with less risk-averse agents. For economies populated by agents (weakly) more risk-averse than log, we provide sufficient conditions for the occurrence of the WOC and for the selffulfilling property of the consensus accuracy to occur that takes agents' risk attitudes into consideration.

Throughout the paper, we use simulations for illustrative purposes; their length varies to accommodate the different convergence rates; to ease comparison, we use the same typical path for all simulations. For the proofs, see the Appendices.

\subsection{Related literature}

A very influential stream of literature argues that asset prices are accurate because financial markets are an efficient aggregator of private information (Grossman 1976, 1978, Radner 1979, Grossman and Stiglitz 1980). Closely related to the literature on information transmission (Aumann 1976, Geanakoplos and Polemarchakis 1982), this literature assumes typically that agents disagree solely due to differences in their private information and provides conditions under which the price formation mechanism reveals all private information to all agents in the market. Because all agents have a common prior, agree on the way to interpret information, and prices instantaneously reveal all available information, in equilibrium all agents must hold the same beliefs and no WOC or selection based on belief heterogeneity can occur.

An alternative explanation for market accuracy, the MSH, relies on the evolutionary argument that markets become accurate because they select for accurate agents 
(Alchian 1950, Friedman 1953). According to the MSH, agents with inaccurate beliefs lose their wealth to accurate agents and, eventually, equilibrium prices are accurate because they reflect only the beliefs of the most accurate agent in the economy (Sandroni 2000). In these models, the market identifies the best model but does not work as an aggregator. By selecting for a unique most accurate agent, the market "destroys" all the accuracy gains that could be achieved by pooling the diverse opinions of the agents who vanish and no WOC can occur. Accordingly, market prices can only be as accurate as the most accurate agent (Blume and Easley 2009), even in knife-edge cases in which there are multiple survivors (Jouini and Napp 2011, Massari 2013). In addition to our model, others in the market selection literature allow for long-run survival of agents with heterogeneous beliefs, but do not explicitly analyze the accuracy of the resulting prices. Survival of agents with heterogeneous beliefs occurs in economies with incomplete markets (Beker and Chattopadhyay 2010, Cogley et al. 2013, Cao 2017), ambiguous averse agents (Guerdjikova and Sciubba 2015), exogenous saving rules (Bottazzi and Dindo 2014, Bottazzi et al. 2018), and recursive preferences (Borovička 2020, Dindo 2019). A model that merges elements of rational learning from prices and selection is Mailath and Sandroni (2003). This model does not endogenously generate the WOC because long-run heterogeneity is a consequence of the presence of noise traders.

Finally, the WOC argument (initially proposed by Galton (1907) and more recently popularized by Surowiecki (2005)) hypothesizes that asset prices are accurate because the opposite, idiosyncratic errors of individual agents are averaged out by the price formation mechanism. The WOC hypothesis has inspired a growing interest in prediction markets (Wolfers and Zitzewitz 2004, Arrow et al. 2008) and social trading platforms (Chen et al. 2014, Breitmayer et al. 2019). Within the prediction markets literature, most of the attention has been focused on static settings. However, there is no solid foundation to justify the WOC argument. The WOC can occur only if the joint distribution of consumption shares and beliefs is such that individual mistakes cancel out. The main limitation of the WOC is the lack of theoretical arguments supporting this assumption (Ali 1977, Manski 2006). Further, even if agents had heterogeneous priors and were rationally processing unbiased signals, the aggregate beliefs might be biased nonetheless due to wealth effects (Ottaviani and Sørensen 2015). Works that also combine dynamic elements such as ours in prediction markets are Kets et al. (2014) and Bottazzi and Giachini (2019). The WOC has also been investigated within other contexts. In the literature of social learning in networks, Golub and Jackson (2010) and Jadbabaie et al. (2012) provide conditions under which agents imitating each other and naively updating their beliefsusing a rule similar to ours-can achieve the same outcome as rational learning models. In the literature on collective problem-solving, Hong and Page (2004) explore the tradeoff between opinion diversity and the difficulty in identifying optimal solutions (see also Page 2007).

\section{THE MODEL}

Time is discrete, indexed by $t$, and begins at date $t=0$. In each period $t \geq 1$, the economy can be in one of $S$ mutually exclusive states, $\mathcal{S}$. The set of partial histories until $t$ is 
the Cartesian product $\Sigma^{t}=x^{t} \mathcal{S}$ and the set of all paths is $\Sigma:=x^{\infty} \mathcal{S} . \sigma=\left(\sigma_{1}, \ldots\right)$ is a representative path, $\sigma^{t}=\left(\sigma_{1}, \ldots, \sigma_{t}\right)$ is a partial history until period $t$, and $\mathcal{F}_{t}$ is the $\sigma$ algebra generated by the cylinders with base $\sigma^{t}$. By construction, $\left(\mathcal{F}_{t}\right)_{t=0}^{\infty}$ is a filtration and $\mathcal{F}$ is the $\sigma$-algebra generated by their union.

$P$ denotes the true measure on $(\Sigma, \mathcal{F})$. In particular, we assume that states of nature are i.i.d. so that the one-step-ahead true probability $P_{t}$ is constant for all $t \geq 1$. With abuse of notation, we denote with $P \in \Delta^{|\mathcal{S}|}$ also such measure.

For any probability measure $\rho$ on $(\Sigma, \mathcal{F}), \rho\left(\sigma^{t}\right):=\rho\left(\left\{\sigma_{1} \times \cdots \times \sigma_{t} \times S \times S \times \cdots\right\}\right)$ is the marginal probability of the partial history $\sigma^{t}$ while $\rho_{t}:=\rho\left(\sigma_{t} \mid \sigma^{t-1}\right)=\rho\left(\sigma^{t}\right) / \rho\left(\sigma^{t-1}\right)$ is the conditional probability of the generic state $\sigma_{t}$ given $\sigma^{t-1}$, so that $\rho\left(\sigma^{t}\right)=$ $\prod_{\tau=1}^{t} \rho\left(\sigma_{\tau} \mid \sigma^{\tau-1}\right)$.

Next, we introduce a number of economic variables with time index $t$. All of these variables are adapted to the information filtration $\left(\mathcal{F}_{t}\right)_{t=0}^{\infty}{ }^{1}$

The economy contains a finite set of agents $\mathcal{I}$. For all paths $\sigma$, each agent $i \in \mathcal{I}$ is endowed with a stream of the consumption good, $\left(e_{t}^{i}(\sigma)\right)_{t=0}^{\infty}$. We take the consumption good in $t=0$ as the numéraire of the economy. Each agent's objective is to maximize the stream of discounted expected utility he gets from consumption. Expectations are computed according to agents' beliefs $p^{i}$, a measure on $(\Sigma, \mathcal{F})$. Beliefs are heterogeneous and agents agree to disagree. Beliefs may be endogenous in that they may rely on a market consensus (see Definition 4, Section 2.2). Naming $q\left(\sigma^{t}\right)$ the date $t=0$ price of the asset that delivers one unit of consumption in event $\sigma^{t}$ and none otherwise, agent $i$ maximization reads:

$$
\max _{\left(c_{t}^{i}(\sigma)\right)_{t=0}^{\infty}} \mathrm{E}_{p^{i}}\left[\sum_{t=0}^{\infty} \beta^{i^{t}} u^{i}\left(c_{t}^{i}(\sigma)\right)\right] \text { s.t. } \sum_{t \geq 0} \sum_{\sigma^{t} \in \Sigma^{t}} q\left(\sigma^{t}\right)\left(c_{t}^{i}(\sigma)-e_{t}^{i}(\sigma)\right) \leq 0 .
$$

In Appendix C, we give the formal definition of the competitive equilibrium when agents' beliefs depend on the endogenous consensus and prove its existence. A competitive equilibrium is a sequence of prices and, for each agent, beliefs and a consumption plan that is preference maximal on the budget set, and such that markets clear in every period: for all $(t, \sigma), \sum_{i \in \mathcal{I}} e_{t}^{i}(\sigma)=\sum_{i \in \mathcal{I}} c_{t}^{i}(\sigma)$. Assumptions Al-A4 below are standard in the market selection literature: $\mathrm{A} 1-\mathrm{A} 3$ ensure the existence of a competitive equilibrium, while A4 guarantees that the market selects for the most accurate agent(s) rather than for those that save the most.

A1 For all agents $i \in \mathcal{I}$, the utility $u^{i}: \mathbb{R}_{+} \rightarrow[-\infty,+\infty]$ is $C^{1}$, strictly concave, increasing, and satisfies the Inada condition at 0 ; that is, $\lim _{c \searrow_{0}} u^{i \prime}(c)=\infty$.

A2 The aggregate endowment is uniformly bounded from above and away from 0 :

$$
\infty>F=\sup _{t, \sigma} \sum_{i \in \mathcal{I}} e_{t}^{i}(\sigma) \geq \inf _{t, \sigma} \sum_{i \in \mathcal{I}} e_{t}^{i}(\sigma)=f>0 .
$$

${ }^{1}$ Whenever there is no ambiguity about the path in question, adapted variables have only the index $t$, so that $x_{t}=x_{t}(\sigma)$. 
A3 (i) For all agents $i \in \mathcal{I}$ and for all $(t, \sigma), p^{i}\left(\sigma^{t}\right)>0 \Leftrightarrow P\left(\sigma^{t}\right)>0$.

(ii) $\exists \epsilon>0$ such that for all agents $i \in \mathcal{I}$ and for all $(t, \sigma), p^{i}\left(\sigma_{t} \mid \sigma^{t-1}\right)>\epsilon .^{2}$

A4 All agents have common discount factor: $\forall i \in \mathcal{I}, \beta^{i}=\beta \in(0,1)$.

\subsection{Agents' accuracy and survival}

In this section, we remind the reader of standard definitions and results from the market selection literature. The asymptotic fate of an agent is characterized by his consumption share as follows.

Definition 1. Agent $i$ vanishes if $\lim _{t \rightarrow \infty} c_{t}^{i}(\sigma)=0, P$-a.s., he survives if $\limsup \operatorname{su}_{t \rightarrow \infty} c_{t}^{i}(\sigma)>0, P$-a.s., he dominates if $\lim _{t \rightarrow \infty} c_{t}^{i}(\sigma) / \sum_{j \in \mathcal{I}} c_{t}^{j}(\sigma)=1, P$-a.s.

Since it became the standard after Blume and Easley (1992), we rank agents' accuracy according to their average (conditional) relative entropies (Kullback-Leibler divergences).

Definition 2. The average relative entropy from $p^{i}$ to the true probability $P$ is

$$
\begin{aligned}
\bar{d}\left(P \| p^{i}\right):= & \lim _{t \rightarrow \infty} \frac{1}{t} \sum_{\tau=1}^{t} d\left(P \| p_{\tau}^{i}\right), \\
& \text { where, for all } \tau, d\left(P \| p_{\tau}^{i}\right):=\mathrm{E}_{P}\left[\ln \frac{P\left(\sigma_{\tau}\right)}{p^{i}\left(\sigma_{\tau} \mid \sigma^{\tau-1}\right)}\right] .
\end{aligned}
$$

The relative entropy is uniquely minimized at $p_{\tau}^{i}=P$, strictly convex, and $d(P \| \pi)=$ $\bar{d}(P \| \pi), P$-a.s. whenever $P$ and $\pi$ are i.i.d. measures. We say the following.

Definition 3. Agent $i$ is more accurate than agent $j$ if $\bar{d}\left(P \| p^{i}\right)<\bar{d}\left(P \| p^{j}\right), P$-a.s. Agent $i$ is as accurate as agent $j$ if $\bar{d}\left(P \| p^{i}\right)=\bar{d}\left(P \| p^{j}\right), P$-a.s.

This notion of accuracy is commonly adopted in the market selection literature because of its straightforward implications for agents' survival. Under A1-A4, the pairwise comparison of agents' accuracies delivers a sufficient condition for an agent to vanish.

Proposition 1 (Sandroni (2000)). Under Al-A4, agent $i$ vanishes if there exists an agent $j \in \mathcal{I}$ who is more accurate:

$$
\bar{d}\left(P \| p^{j}\right)<\bar{d}\left(P \| p^{i}\right), \quad P \text {-a.s. } \Rightarrow \text { Agent i vanishes. }
$$

This fundamental result, together with known results in probability theory, allows us to characterize survival of agents with exogenous beliefs. The difficulty we have to overcome is to calculate the accuracy of agents whose beliefs depend on an endogenous measure of consensus.

\footnotetext{
${ }^{2}$ Lemma 4 in Appendix A guarantees that the endogenous beliefs in our model satisfy A3 on every equilibrium path.
} 


\subsection{Agents' beliefs}

We assume that agents in our economy either have exogenous beliefs or form beliefs for next-period states by giving constant weight to two different models. The first model, $p^{C}$, is endogenous and represents the market consensus; see Section 2.4. The second model, dogmatic probabilities $\left(\pi^{i}\right)$, is exogenous and agent-specific. ${ }^{3}$ We assume that dogmatic probabilities are constant over time ${ }^{4}$ and in the strict interior of the simplex, which ensures that A3 holds (Lemma 4 in Appendix A).

Definition 4. The beliefs of each agent $i \in \mathcal{I}$ are either exogenous such that A3 holds and $\bar{d}(P \| \cdot)$ exists; or given by

$$
\forall(t, \sigma), \quad p^{i}\left(\sigma_{t} \mid \sigma^{t-1}\right)=\left(1-\alpha^{i}\right) p^{C}\left(\sigma_{t} \mid \sigma^{t-1}\right)+\alpha^{i} \pi^{i}\left(\sigma_{t}\right)
$$

with $\alpha^{i} \in(0,1)$ and $\pi^{i} \in \Delta^{S}$ strictly positive.

This rule describes the attitude of an agent who partially believes that markets are accurate. The parameter $\alpha^{i}$ determines how much agent $i$ believes in the accuracy of the consensus. Having exogenous beliefs, equivalently $\alpha^{i}=1$, represents the extreme scenario in which agent $i$ ignores the consensus. This is the standard case in the market selection literature, where it is typically assumed that agents' beliefs are independent of each other and of equilibrium quantities. Whereas $\alpha^{i}=0$ represents the case in which agent $i$ does not give any weight to his dogmatic probabilities because he is certain that markets are accurate - with a similar attitude to the economist who finds a $\$ 20$ bill lying on the ground and refuses to believe it. The intermediate cases of $\alpha^{i} \in(0,1)$ are those that generate the most interesting results. ${ }^{5}$

Definition 4 describes a mental attitude that is consistent with known biases including anchoring (Shiller 1999) and herding (Lakonishok et al. 1992). Furthermore, the beliefs formation rule of Definition 4 has been used to discuss the effect of agents' partial learning from equilibrium prices in the context of static prediction markets (Manski 2006); a similar rule is used in the learning literature on networks (Jadbabaie et al. 2012, Golub and Jackson 2010); and beliefs (1) determine a portfolio that (assuming log utility) coincides with the fractional Kelly rule proposed by MacLean et al. (2011) in the portfolio theory literature.

\footnotetext{
${ }^{3}$ The heterogeneity of dogmatic probabilities is taken as given and we are agnostic about its source. Although all agents receive the same public information $\left(\mathcal{F}_{t}\right)$, on which they trade, they could use it to learn on different models or they could learn on the same models but augment the public information with different private signals.

${ }^{4}$ All results generalize verbatim to the case in which the $\pi^{i}$ probabilities evolve according to Bayes' rule. Agents who learn their dogmatic beliefs via Bayes' rule can be treated WLOG as agents with constant dogmatic beliefs because, in i.i.d. settings, the Bayesian posterior converges generically to a unique model (the model with the lowest K-L divergence to the truth, Berk (1966)) and our measure of accuracy (Definition 3) is an average measure.

${ }^{5}$ We rule out $\alpha^{i}=0$ because $\alpha^{i}=0$ for all $i \in \mathcal{I}$ leads to an indeterminate equilibrium.
} 


\subsection{A definition of the wisdom of the crowd}

We say that the $\mathrm{WOC}^{C}$ occurs if the market consensus, $p^{C}$, is more accurate than the beliefs of the most accurate agent in isolation. Two probabilities play a special role in our definition: the Best Individual Probability $\left(\pi^{\mathrm{BIP}}\right)$, which is the most accurate dogmatic probability, and the Best Collective Probability $\left(\pi^{\mathrm{BCP}}\right)$, which is the most accurate combination of agents' dogmatic probabilities. Moreover, we say that dogmatic probabilities are diverse when the Best Collective Probability differs from the Best Individual Probability, that is, if it is possible to combine dogmatic probabilities into a prediction that is more accurate than that of all dogmatic probabilities.

\section{Definition 5. Given a set of dogmatic probabilities $\left\{\pi^{1}, \ldots, \pi^{I}\right\}$ :}

- the Best Individual Probability is $\pi^{\mathrm{BIP}}=\operatorname{argmin}_{\pi \in\left\{\pi^{1}, \ldots, \pi^{I}\right\}} \bar{d}(P \| \pi)$;

- the Best Collective Probability is $\pi^{\mathrm{BCP}}=\operatorname{argmin}_{p \in \operatorname{Conv}\left(\pi^{1}, \ldots, \pi^{I}\right)} \bar{d}(P \| p)$;

- Agents' beliefs are diverse if it is possible to achieve accuracy gains by balancing the different opinions of market participants: $\pi^{\mathrm{BIP}} \neq \pi^{\mathrm{BCP}}$.

Given our definitions of agents' beliefs and consensuses (below), when an agent is alone in the market his beliefs, his dogmatic probabilities and the consensus coincide $\left(p^{i}=\pi^{i}=p^{C}\right)$. Therefore, we can define the WOC as follows.

Definition 6. The $W O C^{C}$ occurs if $p^{C}$ is more accurate than $\pi^{\mathrm{BIP}}$ :

$$
\bar{d}\left(P \| p^{C}\right)<\bar{d}\left(P \| \pi^{\mathrm{BIP}}\right), \quad P \text {-a.s. }
$$

To gain intuition, consider a two-state, $\mathcal{S}=\{u, d\}$, two-agent, $\mathcal{I}=\{1,2\}$, economy. The true probability of state $u$ is $P(u)=0.5$. Agent 1 is pessimistic about $u$, while agent 2 is optimistic. Their dogmatic probabilities are $\pi^{1}(u)=0.4$ and $\pi^{2}(u)=0.7$, respectively. Clearly, agent 1 has the most accurate dogmatic probabilities, thus $\pi^{\mathrm{BIP}}=\pi^{1}=0.4$, while the most accurate way to combine the dogmatic probabilities of the two agents is $(2 / 3) \pi^{1}(u)+(1 / 3) \pi^{2}(u)=p^{\mathrm{BCP}}=P$. The WOC occurs if market probabilities are more accurate than the dogmatic probability of agent 1 (and thus 2) -in other words, if the market consensus is more accurate than all market participants in isolation.

\subsection{Market consensuses}

A crucial point of our analysis is the definition of the market consensus $p^{C}$. We conduct our analysis using different measures of consensus. The rationale behind these measures is that they weight more the beliefs of agents with larger consumption shares than those of agents with smaller consumption shares, so that the consensus obtained in an economy with a unique agent coincides with his beliefs. All the measures of consensus we propose coincide in economies with constant aggregate endowment in which all agents have log utility. However, under more general assumptions they are not the 
same because they are differently affected by agents' risk attitudes and fluctuations of the aggregate endowment.

The first measure of consensus we propose is market probabilities: $p^{M}$.

Definition 7. For all $(t, \sigma)$, market probabilities are

$$
p^{M}\left(\sigma_{t} \mid \sigma^{t-1}\right)=\sum_{i \in \mathcal{I}} p^{i}\left(\sigma_{t} \mid \sigma^{t-1}\right) \frac{\bar{c}_{t-1}^{i}}{\sum_{j \in \mathcal{I}} \bar{c}_{t-1}^{j}},
$$

where $\bar{c}_{t}^{i}=1 / u^{i \prime}\left(c_{t}^{i}(\sigma)\right)$.

If all agents have log utility and the aggregate endowment is constant, $p^{M}$ coincides with the risk-neutral probabilities and can be calculated from equilibrium prices alone. In these economies, Rubinstein (1974) shows that a representative agent exists and that his unconditional beliefs are $\sum_{i \in \mathcal{I}} p^{i}\left(\sigma^{t}\right) c_{0}^{i} / \sum_{j \in \mathcal{I}} c_{0}^{j}$. Lemma 1 shows that $p^{M}$ makes the analysis of general economies qualitatively equivalent to that of a log economy with no aggregate risk, albeit a distortion of the initial weights.

Lemma 1. Under A1-A4, on a competitive equilibrium for all $(t, \sigma)$ it holds

$$
p^{M}\left(\sigma^{t}\right)=\sum_{i \in \mathcal{I}} p^{i}\left(\sigma^{t}\right) \frac{\bar{c}_{0}^{i}}{\sum_{j \in \mathcal{I}} \bar{c}_{0}^{j}} .
$$

For the general case, the calculation of $p^{M}$ requires knowledge of the preferences and the consumption shares of all agents. While it is unlikely that an agent in the market would have this degree of information, we use market probabilities (equivalently log utility for all agents and constant aggregate endowment) to set a benchmark for the results that follow.

Next, we propose measures of consensus that can be easily calculated from equilibrium prices, also beyond the log utility case. When the aggregate endowment is constant, we study the occurrence of the WOC when some of the agents use the risk-neutral probabilities for consensus.

Definition 8. For all $(t, \sigma)$, the risk-neutral consensus is

$$
p^{\mathrm{RN}}\left(\sigma_{t} \mid \sigma^{t-1}\right)=\frac{q\left(\sigma_{t} \mid \sigma^{t-1}\right)}{\sum_{\tilde{\sigma}_{t}} q\left(\tilde{\sigma}_{t} \mid \sigma^{t-1}\right)},
$$

where $q\left(\sigma_{t} \mid \sigma^{t-1}\right):=q\left(\sigma^{t}\right) / q\left(\sigma^{t-1}\right)$ is the equilibrium price of a claim that pays a unit of consumption at period/event $\sigma_{t}$, in terms of consumption at period/event $\sigma^{t-1}$.

The analysis of economies in which agents rely on the risk-neutral consensus is more complex than it is for agents using $p^{M}$ because agents' risk attitudes do affect 
$p^{\mathrm{RN}}$ accuracy, and thus agents' accuracy and survival. We show that, ceteris paribus, economies with more risk-averse agents generate more accurate risk-neutral probabilities than economies with less risk-averse agents and the WOC $^{\mathrm{RN}}$ occurs under weaker conditions. Lemma 2 expresses the equilibrium value of $p_{t}^{\mathrm{RN}}$ in a way that facilitates its comparison to $p_{t}^{M}$.

Lemma 2. Under A1-A4, on a competitive equilibrium for all $(t, \sigma)$ it holds

$$
p^{\mathrm{RN}}\left(\sigma_{t} \mid \sigma^{t-1}\right) \propto \sum_{i \in \mathcal{I}} p^{i}\left(\sigma_{t} \mid \sigma^{t-1}\right) \frac{\bar{c}_{t-1}^{i}}{\sum_{j \in \mathcal{I}} \bar{c}_{t}^{j}} .
$$

The difference between $p^{M}$ and $p^{\mathrm{RN}}$ becomes apparent in comparing the weights given to agents' beliefs in Definitions 7 with those in Lemma 2: $\bar{c}_{t-1}^{i} / \sum_{j \in \mathcal{I}} \bar{c}_{t-1}^{j} \neq$ $\bar{c}_{t-1}^{i} / \sum_{j \in \mathcal{I}} \bar{c}_{t}^{j}$. The first one is state independent because the ratio involves the marginal utility of consumption in the same period. The second one is state dependent because the ratio compares marginal utilities in two different periods. Moreover, only $p^{\mathrm{RN}}$ requires to be normalized.

In an economy with a unique agent and constant aggregate endowment, both measures satisfy our desiderata to be an unbiased estimator of the beliefs of the agent. However, $p^{\mathrm{RN}}$ fails to satisfy this property in economies where the aggregate endowment varies because there are some $(t, \sigma)$ such that $\bar{c}_{t} \neq \bar{c}_{t-1}$. The last measure of market consensus we study can be calculated from prices and aggregate endowment alone and corrects for this bias in economies in which all agents have common CRRA utility function $u(c)=\left(c^{1-\gamma}-1\right) /(1-\gamma)$.

Definition 9. For all $(t, \sigma)$, the $\gamma$-adjusted risk-neutral consensus is

$$
p_{\gamma}^{\mathrm{RN}}\left(\sigma_{t} \mid \sigma^{t-1}\right)=\frac{q\left(\sigma_{t} \mid \sigma^{t-1}\right) \boldsymbol{e}_{t}(\sigma)^{\gamma}}{\sum_{\tilde{\sigma}_{t}} q\left(\tilde{\sigma}_{t} \mid \sigma^{t-1}\right) \boldsymbol{e}_{t}(\tilde{\sigma})^{\gamma}},
$$

where $\boldsymbol{e}_{t}(\sigma)=\sum_{i \in \mathcal{I}} e_{t}^{i}(\sigma)$ is the aggregate endowment.

Lemma 3 expresses the equilibrium value of $p_{\gamma}^{\mathrm{RN}}$ in economies in which all agents have identical CRRA utilities in a way that facilitates its comparison with $p^{\mathrm{RN}}$ and $p^{M}$. It shows that $p_{\gamma}^{\mathrm{RN}}$ is immune to biases due to fluctuations of the aggregate endowment because it is a consumption share version of the $p^{\mathrm{RN}}$ consensus.

Lemma 3. Under A1-A4, if all agents have common CRRA utility with parameter $\gamma \in$ $(0, \infty)$, on a competitive equilibrium for all $(t, \sigma)$ it holds that

$$
p_{\gamma}^{\mathrm{RN}}\left(\sigma_{t} \mid \sigma^{t-1}\right) \propto \sum_{i \in \mathcal{I}} p^{i}\left(\sigma_{t} \mid \sigma^{t-1}\right) \frac{\phi_{t-1}^{i}(\sigma)^{\gamma}}{\sum_{j \in \mathcal{I}} \phi_{t}^{j}(\sigma)^{\gamma}} ;
$$

where $\phi_{t}^{i}(\sigma)=c_{t}^{i}(\sigma) / \sum_{j \in \mathcal{I}} c_{t}^{j}(\sigma)$. 


\section{Main Results Related to $p^{M}$}

In this section, we characterize the accuracy of $p^{M}$, provide necessary conditions and sufficient conditions for the $\mathrm{WOC}^{M}$ to occur, and demonstrate its self-fulfilling property. If a diverse group of agents believes in the accuracy of $p^{M}$, market probabilities are indeed accurate.

\subsection{Accuracy of $p^{M}$}

Here, we provide bounds on the relative accuracy of $p^{M}$ with respect to that of agents' beliefs. Proposition 2 characterizes the relative accuracy of $p^{M}$ with respect to that of agents without solving for the equilibrium and independently of how agents form their beliefs. It shows that the dynamics of equilibrium consumption shares is such that we have the following.

Proposition 2. Under Al-A4,

(i) no agent can be more accurate than $p^{M}$ :

$$
\forall i \in \mathcal{I}, \quad \bar{d}\left(P \| p^{i}\right) \geq \bar{d}\left(P \| p^{M}\right), \quad P \text {-a.s.; }
$$

(ii) agent $i$ survives only if he is as accurate as $p^{M}$ :

$$
\text { Agent i survives } \Rightarrow \bar{d}\left(P \| p^{i}\right)=\bar{d}\left(P \| p^{M}\right), \quad P \text {-a.s. }
$$

Proposition 2 simplifies our analysis because standard techniques to approximate market probabilities and the accuracy of agents' beliefs cannot be used when agents' beliefs depend on the endogenous consensus. All the results in this section are obtained by combining Propositions 1 and 2, and by taking advantage of the convexity of the relative entropy.

Next, Proposition 3 shows that market probabilities provide a fundamental hedging benefit to the agents. By believing in $p^{M}$ an agent weakly improves its accuracy irrespectively of his dogmatic beliefs, of the beliefs of the other agents, and of the true probability.

Proposition 3. Under A1-A4, if $\alpha^{i} \in(0,1)$ and $i$ uses $p^{M}$ for consensus,

$$
\bar{d}\left(P \| p^{i}\right) \leq \bar{d}\left(P \| \pi^{i}\right), \quad P \text {-a.s. }
$$

with strict inequality if there exists an $\epsilon>0$ such that $\left\|p_{t}^{M}-\pi^{i}\right\|>\epsilon$ a positive fraction of periods.

If $\pi^{i}$ is the true model, agent $i$ 's average accuracy is not diminished by mixing with market probabilities because market probabilities converge to $\pi^{i}$ exponentially fast since he dominates. Otherwise, if agent $i$ 's subjective probabilistic model of the world is incorrect, mixing with the consensus improves agent $i$ 's accuracy whenever the consensus is different from his dogmatic beliefs because Proposition 2 guarantees that the consensus is more accurate than $\pi^{i}$. 
Furthermore, $p^{M}$ is at least as accurate as $\pi^{\mathrm{BIP}}$ and at most as accurate as $\pi^{\mathrm{BCP}}$, provided that all agents with $\alpha^{i} \in(0,1)$ use $p^{M}$ for consensus.

Corollary 1. Under A1-A4, if all agents with $\alpha^{i} \in(0,1)$ use $p^{M}$ for consensus, $p^{M}$ is at least as accurate as $\pi^{\mathrm{BIP}}$ and at most as accurate as $\pi^{\mathrm{BCP}}$ :

$$
\bar{d}\left(P \| \pi^{\mathrm{BCP}}\right) \leq \bar{d}\left(P \| p^{M}\right) \leq \bar{d}\left(P \| \pi^{\mathrm{BIP}}\right), \quad P \text {-a.s. }
$$

Proof. $\bar{d}\left(P \| p^{M}\right) \quad \leq^{\text {By Prop. } 2} \quad \bar{d}\left(P \| p^{\text {BIP }}\right) \quad \leq$ By Prop. $3 \quad \bar{d}\left(P \| \pi^{\text {BIP }}\right) . \quad \quad \bar{d}\left(P \| p^{M}\right) \geq$ $\bar{d}\left(P \| \pi^{\mathrm{BCP}}\right)={ }^{P \text {-a.s. }} \min _{p \in \operatorname{Conv}\left(\pi^{1}, \ldots, \pi^{I}\right)} d(P \| p)$ because $\forall(t, \sigma), p_{t}^{M} \in \in^{\text {By Lem. } 5} \operatorname{Conv}\left(\pi^{1}, \ldots\right.$, $\left.\pi^{I}\right)$.

Corollary 1 is proven showing that in the long run either the agent with the most accurate dogmatic probabilities dominates, and market probabilities are as accurate as $\pi^{\mathrm{BIP}}$, or there is long-run heterogeneity, and market probabilities are a convex combination of the surviving agents' dogmatic probabilities-thus, at most as accurate as $\pi^{\mathrm{BCP}}$ by definition.

\subsection{Necessary conditions for the WOC ${ }^{M}$}

When the reference consensus is $p^{M}$, we identify two necessary conditions for the WOC $^{M}$. First, it must be possible to achieve accuracy gains by balancing the different opinions of market participants (diversity). Second, at least some of the agents must believe in market accuracy —which is necessary for long-run heterogeneity. Selection forces can induce a nondegenerate joint distribution of consumption shares and beliefs that makes market probabilities more accurate than the most accurate agent in isolation only if the economy is diverse and some beliefs are endogenous.

Proposition 4. Under A1-A4, if all agents use $p^{M}$ for consensus, the WOC ${ }^{M}$ can occur only if beliefs are diverse and the beliefs of at least one agent depend on $p^{M}$.

The first requirement (diversity) tells us that the $\mathrm{WOC}^{M}$ cannot occur if all agents share the same bias. For example, in an economy with two states in which all dogmatic probabilities overweight the same state, no $\mathrm{WOC}^{M}$ can occur because the most accurate combination of agents' beliefs is the one obtained by giving all wealth to the least biased among the agents (BIP). Furthermore, this condition tells us that the WOC $^{M}$ cannot occur if there is an agent who knows (or eventually learns) the truth because $P=\pi^{\mathrm{BCP}}=\pi^{\mathrm{BIP}}$.

The second requirement (relevance of the market consensus) confirms the standard result in the selection literature that the WOC cannot occur when agents' beliefs do not depend on endogenous quantities. For example, suppose the market has an optimistic and a pessimistic agent. If the pessimistic agent is less accurate than the optimist, then the pessimist vanishes, market probabilities reflect only the beliefs of the optimist and no WOC occurs (Blume and Easley 2009). 

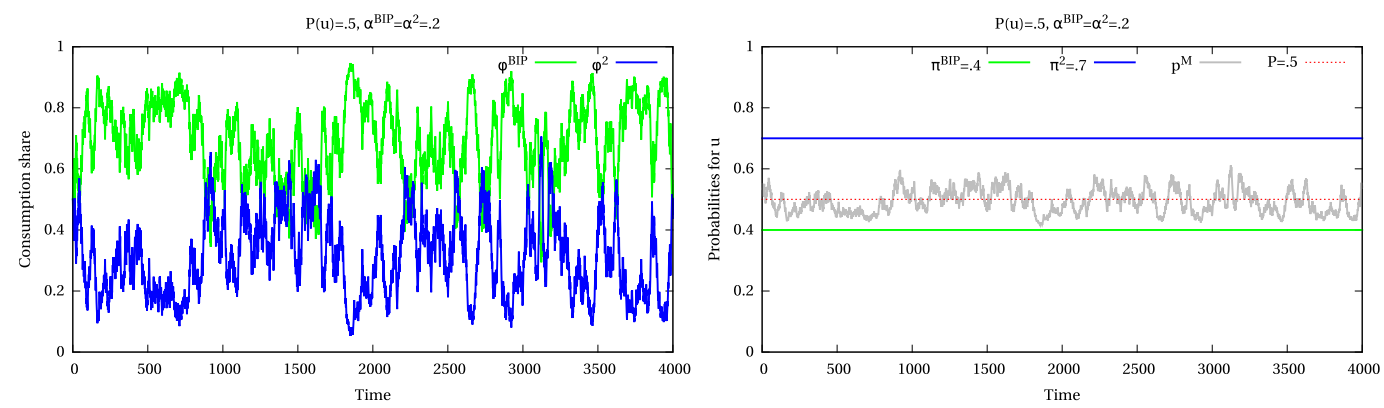

Figure 1. Consumption shares [left] and market probabilities [right] dynamics in a two-state log economy with dogmatic beliefs $\left[\pi^{\mathrm{BIP}}(u), \pi^{2}(u)\right]=[0.4,0.7]$ and mixing coefficient $\left[\alpha^{\mathrm{BIP}}, \alpha^{2}\right]=[0.2,0.2]$. Both conditions of Proposition 5 hold and the WOC ${ }^{M}$ occurs. Consumption shares never find a resting point, and market probabilities are more accurate than $\pi^{\mathrm{BIP}}$.

\subsection{Sufficient conditions for the $W O C^{M}$}

While the market might be populated by many agents with arbitrary beliefs and preferences, the next condition shows that to guarantee that the $\mathrm{WOC}^{M}$ occurs it suffices to verify a condition on only two agents. If agent BIP mixes with $p^{M}$ and there is an agent $i$ with $\alpha^{i} \in(0,1)$ that would be more accurate than BIP if BIP were to dominate $\left(p^{M}=\pi^{\mathrm{BIP}}\right.$ ), then at least two agents survive and the $\mathrm{WOC}^{M}$ occurs.

Proposition 5. Under A1-A4, the WOC ${ }^{M}$ occurs and at least two agents survive if agent $\mathrm{BIP}$ relies on $p^{M}$ with $\alpha^{\mathrm{BIP}} \in(0,1)$ and

$$
\exists i \in \mathcal{I}: \quad \bar{d}\left(P \|\left(1-\alpha^{i}\right) \pi^{\mathrm{BIP}}+\alpha^{i} \pi^{i}\right)<\bar{d}\left(P \| \pi^{\mathrm{BIP}}\right) .
$$

Proof. The condition on $p^{i}$ is sufficient to guarantee that agent BIP does not have a consumption share arbitrarily close to one in most periods-otherwise, agent $i$ would be more accurate than agent BIP, violating Proposition 1 . So, $p_{t}^{M} \neq \pi^{\mathrm{BIP}}$ a positive fraction of periods and

$$
\bar{d}\left(P \| p^{M}\right) \leq \text { Prop. } 2 \bar{d}\left(P \| p^{\text {BIP }}\right)<\text { by Prop. } 3 \bar{d}\left(P \| \pi^{\text {BIP }}\right) .
$$

The rationale for these conditions is as follows. Equation (3) guarantees that agent BIP cannot dominate, and $\alpha^{\mathrm{BIP}} \in(0,1)$ guarantees that his beliefs $p^{\mathrm{BIP}}$ are more accurate than his dogmatic beliefs $\pi^{\mathrm{BIP}}$. If agent BIP were to dominate, no $\mathrm{WOC}^{M}$ could occur because $p^{M}$ would be as accurate as his dogmatic beliefs; if agent BIP survived with $\alpha^{\mathrm{BIP}}=1$, then no WOC ${ }^{M}$ could occur because $p^{M}$ would be as accurate as $\pi^{\mathrm{BIP}}$ by Proposition 2. We can focus on agent BIP's fate alone because he is the only agent that could survive in isolation and, if he vanished, the WOC ${ }^{M}$ would occur because $p^{M}$ would be at least as accurate as $p^{\mathrm{BIP}}$ which, by Proposition 3, would be strictly more accurate than $\pi^{\text {BIP }}$.

Figure 1 illustrates Proposition 5 in a log economy with two states, $\mathcal{S}=\{u, d\}$, and two agents $\mathcal{I}=\{\mathrm{BIP}, 2\}$. The true probability of state $u$ is $P(u)=0.5$. Agent BIP is pessimistic about $u$, while agent 2 is optimistic. Their dogmatic probabilities are $\pi^{\mathrm{BIP}}(u)=$ 
0.4 and $\pi^{2}(u)=0.7$, respectively. Because agents' beliefs are diverse $\left(\pi^{\mathrm{BIP}} \neq \pi^{\mathrm{BCP}}=P\right)$ it is possible to achieve accuracy gains by mixing their opinions. With $\alpha^{\mathrm{BIP}}=\alpha^{2}=0.2$, both conditions of Proposition 5 are satisfied. Agents give enough weight to market probabilities to ensure that no agent can dominate in the market. If an agent were to dominate, market probabilities would coincide with his dogmatic beliefs, making the beliefs of the other agent more accurate than his, in contradiction to Proposition 1. Furthermore, $\alpha^{\mathrm{BIP}} \in(0,1)$ guarantees that $p^{M}$ is more accurate than $\pi^{\mathrm{BIP}}$ because it ensures that the $p_{t}^{M}$ s stay closer than $\pi^{\mathrm{BIP}}$ to $P$ in most periods. ${ }^{6}$

Remark. If the economy has two agents, BIP and 2, the two conditions of Proposition 5 are also jointly necessary. If agent 2's beliefs violate equation (3), BIP dominates and no WOC ${ }^{M}$ occurs because $p_{t}^{M} \rightarrow \pi^{\mathrm{BIP}}$. Whereas, if agent BIP does not mix, he survives and no WOC $^{M}$ occurs because $\bar{d}\left(P \| p^{M}\right)=\bar{d}\left(P \| \pi^{\mathrm{BIP}}\right)$ by Proposition 2 . In general, the mixing requirement for agent BIP is not necessary, what is necessary is to have at least two agents with an opposite bias who rely on market probabilities. The stronger result of Theorem 1 (below) makes no assumption on agent BIP's beliefs.

\subsection{Accurate markets: A self-fulfilling prophecy $\left(p^{M}\right)$}

Here, we demonstrate that if there is a group of agents in the economy with beliefs around the truth who are very confident that market probabilities are accurate, then market probabilities are indeed (almost) accurate, irrespective of the beliefs of the other agents. By relying strongly on market probabilities, agents generate a virtuous interaction that makes their beliefs and the market both more accurate. In equilibrium, the selection forces endogenously generate a joint distribution of consumption share and beliefs such that market probabilities are (almost) correct even if no agent knows the truth.

THEOREM 1. Let $\left(\mathcal{E}_{\alpha}\right)$ be a family of economies that satisfies A1-A4 with a subset of agents $\hat{\mathcal{I}}$ that relies on $p^{M}$ with $\alpha^{i} \in(0, \bar{\alpha}]$ and such that $P \in \operatorname{Conv}(\hat{\mathcal{I}})$. Name each economy market probabilities process $\left(p_{t, \bar{\alpha}}^{M}\right)_{t=0}^{\infty}$, then

$$
\lim _{\bar{\alpha} \rightarrow 0} \bar{d}\left(P \| p_{\bar{\alpha}}^{M}\right)=0, \quad P \text {-a.s. }
$$

For intuition, consider a two-state log economy with only two agents, one optimistic, $i^{o}$, and one pessimistic, $i^{p}$. For $\bar{\alpha}$ small enough, both agents survive by Proposition 3 and are equally accurate by Proposition 2. Because $\bar{\alpha}$ is small, their beliefs are almost identical and consumption shares do not change much over time. Accordingly, $p_{t, \bar{\alpha}}^{M}$ must spend most periods close to a value $\hat{p}^{M}(\bar{\alpha})$ that makes the relative entropies $d\left(P \mid p_{t}^{i^{p}}\right)$ and $d\left(P \mid p_{t}^{i^{o}}\right)$ equal. As $\bar{\alpha}$ goes to zero, $\hat{p}^{M}(\bar{\alpha})$ goes to $P$ because any other value would

\footnotetext{
${ }^{6}$ Formally, consumption shares are mean-reverting processes around the value $\bar{\phi}^{\text {BIP }}$ that determines a market probability $\bar{p}^{M}$ which makes agents BIP and 2 have equal relative entropy, i.e., $\phi_{t}^{\text {BIP }} \gtreqless \bar{\phi}^{\text {BIP }} \Leftrightarrow$ $d\left(P \| p_{t}^{\mathrm{BIP}}\right) \gtreqless d\left(P \| p_{t}^{2}\right)$. The WOC ${ }^{M}$ occurs because $\bar{p}^{M}$ is more accurate than $\pi^{\mathrm{BIP}}$ and $\pi^{2}$, and market probabilities stay close to $\bar{p}^{M}$ a large enough fraction of periods.
} 

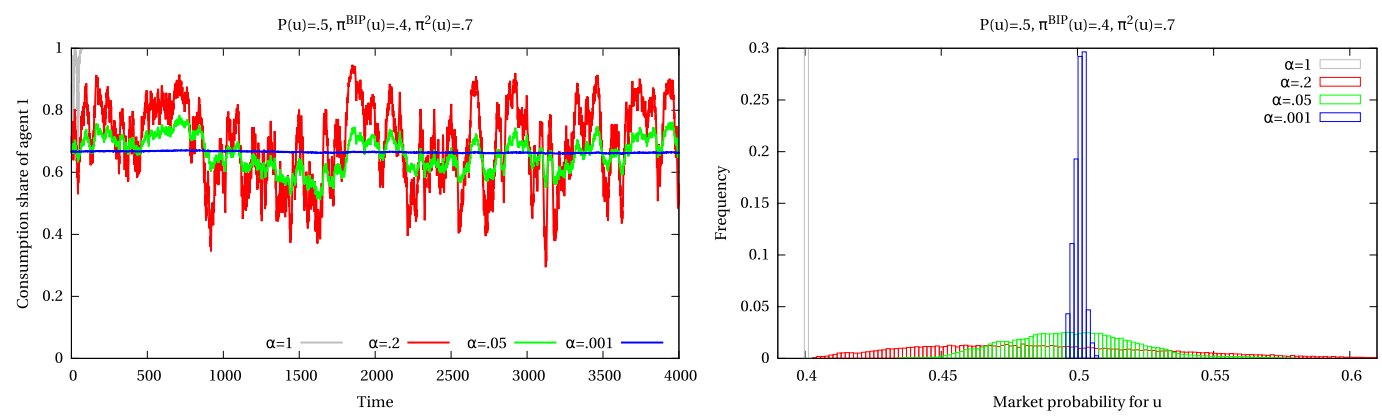

FIgURE 2. Consumption share dynamics [left] and $p^{M}$ frequencies [right] in four log economies with true probability $P(u)=0.5$, two agents with dogmatic probabilities $\pi^{\mathrm{BIP}}(u)=0.4$ and $\pi^{2}(u)=0.7, \alpha^{\mathrm{BIP}}=\alpha^{2}=\bar{\alpha}$ and four different values of $\bar{\alpha}=[1,0.2,0.05,0.001]$. The figure shows that a smaller $\bar{\alpha}$ determines frequencies of $p^{M}$ that are more concentrated around the truth.

make one agent more accurate than the other. For general economies, only agents in $\hat{\mathcal{I}}$ survive and the same intuition applies. ${ }^{7}$

Figure 2 illustrates Theorem 1. It shows [left] the dynamics of consumption shares and [right] the frequency of market probabilities of four economies that differ only in their value of $\bar{\alpha}$. All economies have two agents with dogmatic probabilities $\pi^{\mathrm{BIP}}(u)=$ 0.4 and $\pi^{2}(u)=0.7$, so that $\pi^{\mathrm{BIP}} \neq P \in \operatorname{Conv}\left(\pi^{\mathrm{BIP}}, \pi^{2}\right)$ and $\alpha^{\mathrm{BIP}}=\alpha^{2}=\bar{\alpha}$. As per Proposition 4 , when $\bar{\alpha}=1$, no WOC occurs: prices are as accurate as $\pi^{\mathrm{BIP}}$. As per Proposition 5 , for $\bar{\alpha}$ low enough, no agent dominates and market probabilities are more accurate than $\pi^{\mathrm{BIP}}$. In this specific example, $\bar{\alpha}=0.2$ is already small enough for agent BIP not to dominate. As per Theorem 1 , for $\bar{\alpha}=0.001 \approx 0$ the market probabilities distribution becomes concentrated in a small interval around $P$, which makes $p^{M}$ almost as accurate as the truth. If agents strongly believe that the market is accurate, then the market is indeed accurate.

\section{Main Results Related to $p^{\mathrm{RN}}$ AND $p_{\gamma}^{\mathrm{RN}}$}

In this section, we study the long-run property of markets in which (some) agents use either $p^{\mathrm{RN}}$ or $p_{\gamma}^{\mathrm{RN}}$ for market consensus under the following assumption:

\footnotetext{
${ }^{7}$ Formally, the $p^{M}$ process is characterized by three parameters which depend on $\bar{\alpha}$. These are its drift, its variance, and the threshold, $\hat{p}^{M}$, that determines a drift change. The effect of $\bar{\alpha}$ on $\hat{p}^{M}$ is easy to obtain: $\hat{p}^{M} \rightarrow^{\bar{\alpha} \rightarrow 0} P$. The theorem holds because for every interval around $\hat{p}^{M}, \bar{\alpha}$ can be chosen small enough to ensure that the market belief process spends most of its periods in that interval. The difficulty in proving the result is that a lower $\bar{\alpha}$ implies a lower variance, but also a weaker mean-reverting drift of the market probability process- the selection forces are weaker because agents' beliefs become more similar. Thus, we have to determine which effect dominates when $\bar{\alpha}$ is small. To make things worse, the per-period variances and drifts change over time and are path-dependent. Our result implies that the accuracy gain for a more accurate mean-reverting point and a lower variance of the market probability process more than compensates for the accuracy loss due to weaker mean-reverting forces. Although market probabilities might take a long time to reach $\hat{p}^{M}$ when $\bar{\alpha}$ is small, a low $\bar{\alpha}$ makes $p^{M}$ accurate because it forces $p^{M}$ to remain close to $\hat{p}^{M}$ after reaching it.
} 
A5 Let $\overline{\mathcal{I}}:=\operatorname{BIP} \cup\left\{i \in \mathcal{I}: \alpha^{i} \neq 1\right\}$; either (i) the aggregate endowment is constant and all agents in $\overline{\mathcal{I}}$ have CRRA utility or (ii), the aggregate endowment is not constant and all agents in $\overline{\mathcal{I}}$ have identical CRRA utility. ${ }^{8}$

Because the results we derive under $\boldsymbol{A 5}$ (i) and (ii) are identical, we adopt the abuse of notation $p^{\mathrm{RN}}=p_{\gamma}^{\mathrm{RN}}$ when the aggregate endowment is not constant. ${ }^{9}$

The equilibrium dynamics of an economy in which agents use $p^{\mathrm{RN}}$ for consensus differs from that of an economy in which the same agents use $p^{M}$ for consensus. For example, it is possible that if agents use $p^{\mathrm{RN}}$ for consensus there is a dominating agent while, on the same path $\sigma$, long-run heterogeneity would appear if the same agents were to use $p^{M}$ for consensus. Moreover, $p^{\mathrm{RN}}$ does not satisfy the properties of $p^{M}$ discussed in Section 3: the belief of every surviving agent is typically not as accurate as $p^{\mathrm{RN}}$ (see Proposition 6 , below) and $p_{t}^{\mathrm{RN}}$ might not be a convex combination of agents' dogmatic beliefs.

\subsection{Accuracy of $p^{\mathrm{RN}}$}

In this section, we characterize the relative accuracy of $p^{\mathrm{RN}}$ and $p^{M}$, and discuss its dependence on agents' risk attitudes. Proposition 6 illustrates how the RRA parameters of the surviving agents affect the sign of $\bar{d}\left(P \| p^{\mathrm{RN}}\right)-\bar{d}\left(P \| p^{M}\right)$. Ceteris paribus, economies with more risk-averse agents determine (weakly) more accurate risk-neutral probabilities.

Proposition 6. Under A1-A5, let $\hat{\mathcal{I}}$ be the set of surviving agents, then

(i) $\forall i \in \hat{\mathcal{I}}, \gamma^{i} \in(0,1] \Rightarrow p^{\mathrm{RN}}$ is at most as accurate as $p^{M}$ :

$$
\bar{d}\left(P \| p^{\mathrm{RN}}\right) \geq \bar{d}\left(P \| p^{M}\right), \quad P \text {-a.s. }
$$

(ii) $\forall i \in \hat{\mathcal{I}}, \gamma^{i}=1 \Rightarrow p^{\mathrm{RN}}$ is as accurate as $p^{M}$ :

$$
\bar{d}\left(P \| p^{\mathrm{RN}}\right)=\bar{d}\left(P \| p^{M}\right), \quad P \text {-a.s. }
$$

(iii) $\forall i \in \hat{\mathcal{I}}, \gamma^{i} \in[1, \infty) \Rightarrow p^{\mathrm{RN}}$ is at least as accurate as $p^{M}$ :

$$
\bar{d}\left(P \| p^{\mathrm{RN}}\right) \leq \bar{d}\left(P \| p^{M}\right), \quad P \text {-a.s.; }
$$

where (i) and (iii) hold with strict inequality if and only if there is long-run heterogeneity in beliefs and at least one among the surviving agents has $\alpha \in(0,1)$.

Looking at the proof of Proposition 6 we see that the differential in accuracy between $p^{M}$ and $p^{\mathrm{RN}}$ is due to the equation

$$
\bar{d}\left(P \| p^{\mathrm{RN}}\right)=\bar{d}\left(P \| p^{M}\right)+\lim _{t \rightarrow \infty} \frac{1}{t} \sum_{\tau=1}^{t} \ln \sum_{\tilde{\sigma}_{\tau}} \frac{q\left(\tilde{\sigma}_{\tau} \mid \sigma^{\tau-1}\right)}{\beta}, \quad P \text {-a.s. }
$$

\footnotetext{
${ }^{8}$ The reason why we need only to pose assumptions on agents in $\overline{\mathcal{I}}$ is that Proposition 1 guarantees that the only agent with exogenous beliefs that might survive and have long-run effect on the consensus is agent BIP.

${ }^{9}$ In the Appendix, we present proofs for the two settings separately, when needed.
} 
which allows for the following economic intuition for the difference between $p^{M}$ and $p^{\mathrm{RN}}$ accuracies (see also Massari (2020)). In every $(t-1, \sigma), \sum_{\tilde{\sigma}_{t}} q\left(\tilde{\sigma}_{t} \mid \sigma^{t-1}\right)$ is the cost of moving a unit of consumption for sure a period ahead, i.e., the reciprocal of the riskfree rate. The effect of risk attitudes on the risk-free rate follows this intuition. In every period most agents subjectively believe that assets are mispriced and trade for speculative reasons because they disagree. When agents have log utility $(\gamma=1)$, prices (and thus interest rates) do not affect optimal saving choices (the substitution effect equals the income effect) and the reciprocal of the risk-free rate is given by the discount factor: for all $(t, \sigma), \beta=\sum_{\tilde{\sigma}_{t}} q\left(\tilde{\sigma}_{t} \mid \sigma^{t-1}\right)$. However, if $\gamma<(>) 1$, the substitution effect is stronger (weaker) than the income effect, each agent optimally chooses to save more (less) aggressively than if they had log utility, and a lower (higher) risk-free rate arises: for all $(t, \sigma), \sum_{\tilde{\sigma}_{\tau}} q\left(\tilde{\sigma}_{\tau} \mid \sigma^{\tau-1}\right)>(<) \beta$. When there is heterogeneity in a positive fraction of periods, this effect renders $p^{\mathrm{RN}}$ less (more) accurate than $p^{M}$. In the standard case of exogenous beliefs, this effect is present but either disappears in the short run because one agent dominates, or its magnitude is too small to be captured by an average measure of accuracy (Massari 2017). ${ }^{10}$

\subsection{Sufficient conditions for the $W O C^{\mathrm{RN}}$}

The sufficient conditions for the WOC ${ }^{\mathrm{RN}}$ to occur need to take into account how the risk attitudes of the surviving agents affect $p^{\mathrm{RN}}$ 's accuracy. Proposition 6 tells us that, with general preferences, $p^{\mathrm{RN}}$ might be less accurate than $p^{M}$ or even $\pi^{\mathrm{BIP}}$ in equilibrium. This eventuality makes it harder for the $\mathrm{WOC}^{\mathrm{RN}}$ to occur when agents rely on $p^{\mathrm{RN}}$ rather than $p^{M}$. Stronger conditions are needed to prevent the system from entering a dynamics that has long-run heterogeneity but does not deliver an accurate consensus and our proof technique cannot handle this case. In this paper, we focus on the case in which all agents in $\overline{\mathcal{I}}$ have CRRA utility with $\gamma^{i}>1$ and leave the general case for future research.

If all agents in $\overline{\mathcal{I}}$ have CRRA utility with $\gamma^{i}>1$ Proposition 6 guarantees that $p^{\mathrm{RN}}$ is at least as accurate as $p^{M}$ and the sufficient condition we find is easier to satisfy than that of Proposition 5. Specifically, Proposition 7 does not require agent BIP's beliefs to depend on the consensus.

Proposition 7. Under A1-A5, the WOC ${ }^{\mathrm{RN}}$ occurs and at least two agents survive, if all agents $j \in \overline{\mathcal{I}}$ have CRRA utility with $\gamma^{j}>1$ and

$$
\exists i \in \overline{\mathcal{I}}: \quad \bar{d}\left(P \|\left(1-\alpha^{i}\right) \pi^{\mathrm{BIP}}+\alpha^{i} \pi^{i}\right)<\bar{d}\left(P \| \pi^{\mathrm{BIP}}\right) .
$$

Notably, Proposition 7 holds irrespective of the consensus used by the agents. Figure 3 illustrates Proposition 7. [left] Agent 2 mixes with $p^{\mathrm{RN}}$, for $\gamma=2>1$ and $\left[\alpha^{\mathrm{BIP}}, \alpha^{2}\right]=$ $[1,0.2]$ condition (4) is satisfied, agent BIP cannot dominate and the WOC ${ }^{\mathrm{RN}}$ occurs. [right] The dynamics of $p^{\mathrm{RN}}$ on the same path for an economy with the same parameters but in which agent 2 mixes using $p^{M}$, rather then $p^{\mathrm{RN}}$. As discussed in the remark following Proposition 5, this economy does not generate the WOC $^{M}$ because BIP survives

\footnotetext{
${ }^{10}$ The same effect is present with exogenous beliefs when there is long-run heterogeneity, e.g., with recursive preferences; see Borovička (2020) and Dindo (2019).
} 

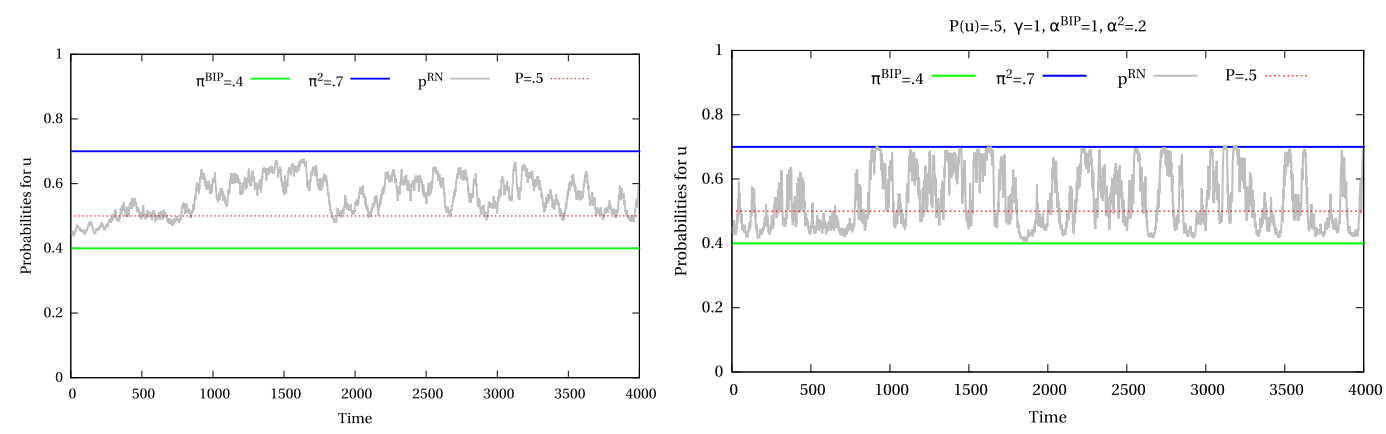

FIgURE 3. [left] $p_{t}^{\mathrm{RN}}$ dynamics in a two-state economy in which agents mix using $p^{\mathrm{RN}}$ with parameters $\left[\pi^{\mathrm{BIP}}(u), \pi^{2}(u)\right]=[0.4,0.7],\left[\alpha^{\mathrm{BIP}}, \alpha^{2}\right]=[1,0.2], \gamma^{\mathrm{BIP}}=2=\gamma^{2}$. [right $p_{t}^{\mathrm{RN}}$ dynamics in an economy with the same parameters in which agent 2 mixes using $p^{M}$, rather than $p^{\mathrm{RN}}$.

but does not mix. Nevertheless, it does generate the WOC $^{\mathrm{RN}}$ because there is long-run heterogeneity so that $p^{\mathrm{RN}}$ is more accurate than $p^{M}$ (Proposition 6) which is at least as accurate as $p^{\mathrm{BIP}}=\pi^{\mathrm{BIP}}$ (Proposition 3).

\subsection{Accurate markets: A self-fulfilling prophecy (RN)}

Here, we give conditions under which the self-fulfilling prophecy discussed in Section 3.4 holds when agents use $p^{\mathrm{RN}}$ for market consensus. As for our sufficient conditions, risk attitudes have an effect on the occurrence of the WOC ${ }^{\mathrm{RN}}$ and we focus exclusively on the case in which all agents in $\overline{\mathcal{I}}$ have CRRA utility with $\gamma^{i} \geq 1$. Under this assumption, the self-fulfilling prophecy condition we derive using $p^{\mathrm{RN}}$ coincides with that of Theorem 1.

THEOREM 2. Let $\left(\mathcal{E}_{\alpha}\right)$ be a family of economies that satisfies A1-A5 with a subset of agents $\hat{\mathcal{I}}$ that relies on $p^{\mathrm{RN}}$ with $\alpha^{i} \in(0, \bar{\alpha}]$ and such that $P \in \operatorname{Conv}(\hat{\mathcal{I}})$. Name each economy risk-neutral probabilities process $\left(p_{t, \bar{\alpha}}^{\mathrm{RN}}\right)_{t=0}^{\infty}$; then, if all agents in $\overline{\mathcal{I}}$ have CRRA utilities,

$$
\forall i \in \overline{\mathcal{I}}, \quad \gamma^{i} \geq 1 \Rightarrow \lim _{\bar{\alpha} \rightarrow 0} \bar{d}\left(P \| p_{\bar{\alpha}}^{\mathrm{RN}}\right)=0, \quad P \text {-a.s. }
$$

\section{Conclusion}

We provide conditions under which the MSH and the WOC can be reconciled in a dynamic economy where agents naively learn from an endogenous measure of consensus. Moreover, we show that if a group of agents strongly believe in market accuracy and their beliefs can be combined to obtain the truth, a virtuous self-fulling prophecy occurs. Although no agent knows the truth, and the initial joint distribution of consumption shares and beliefs might be such that the initial consensus is very inaccurate, market selection forces endogenously generate a joint dynamics of consumption shares and beliefs such that consensuses are almost as accurate as the truth. When agents use the risk-neutral probability for consensus, we show how risk attitudes affect the accuracy of market consensuses and beliefs, and characterize their overall effect on the WOC. 


\section{Appendix A}

In the proofs, we sometimes omit the conditioning notation for prices and probabilities and adopt the more compact notation: for $j \in \mathcal{I} \cup M \cup \mathrm{RN}, p^{j}\left(\sigma_{t} \mid\right):=p^{j}\left(\sigma_{t} \mid \sigma^{t-1}\right)$ and $q\left(\sigma_{t} \mid\right):=q\left(\sigma_{t} \mid \sigma^{t-1}\right)$. Furthermore, we make use of the symbols $\asymp$ and $O(\cdot)$ with the meanings:

$$
\begin{aligned}
& f(x)=O(g(x)) \quad \text { if } \limsup _{x}\left|\frac{f(x)}{g(x)}\right|<\infty, \\
& f(x) \asymp g(x) \text { if } \forall x, f(x)>0, g(x)>0 \text { and }\left\{\begin{array}{l}
\limsup _{x} \frac{f(x)}{g(x)}<\infty, \\
\liminf _{x} \frac{f(x)}{g(x)}>0 .
\end{array}\right.
\end{aligned}
$$

Proof of Lemma 1.

$$
\begin{aligned}
& \forall(t, \sigma), \quad p^{M}\left(\sigma^{t}\right) \\
& =\prod_{\tau=1}^{t} p^{M}\left(\sigma_{\tau} \mid \sigma^{\tau-1}\right) \\
& =\left(\sum_{i \in \mathcal{I}} p^{i}\left(\sigma_{t} \mid \sigma^{t-1}\right) \frac{\bar{c}_{t-1}^{i}(\sigma)}{\sum_{j \in \mathcal{I}} \bar{c}_{t-1}^{j}(\sigma)}\right) \prod_{\tau=1}^{t-1} p^{M}\left(\sigma_{\tau} \mid \sigma^{\tau-1}\right) \\
& ={ }^{(i)}\left(\sum_{i \in \mathcal{I}} p^{i}\left(\sigma_{t} \mid \sigma^{t-1}\right) p^{i}\left(\sigma_{t-1} \mid \sigma^{t-2}\right) \frac{\bar{c}_{t-2}^{i}(\sigma)}{\sum_{j \in \mathcal{I}} \bar{c}_{t-2}^{j}(\sigma)}\right) \frac{1}{p^{M}\left(\sigma_{t-1} \mid \sigma^{t-1}\right)} \prod_{\tau=1}^{t-1} p^{M}\left(\sigma_{\tau} \mid \sigma^{\tau-1}\right) \\
& \quad=\sum_{i \in \mathcal{I}} p^{i}\left(\sigma_{t} \mid \sigma^{t-1}\right) p^{i}\left(\sigma_{t-1} \mid \sigma^{t-2}\right) \frac{\bar{c}_{t-2}^{i}(\sigma)}{\sum_{j \in \mathcal{I}} \bar{c}_{t-2}^{j}(\sigma)} \prod_{\tau=1}^{t-2} p^{M}\left(\sigma_{\tau} \mid \sigma^{\tau-1}\right) \\
& \quad \vdots \\
& \quad=\sum_{i \in \mathcal{I}} \prod_{\tau=1}^{t} p^{i}\left(\sigma_{\tau} \mid \sigma^{\tau-1}\right) \frac{\bar{c}_{0}^{i}}{\sum_{j \in \mathcal{I}} \bar{c}_{0}^{j}} \\
& \quad=\sum_{i \in \mathcal{I}} p^{i}\left(\sigma^{t}\right) \frac{\bar{c}_{0}^{i}}{\sum_{j \in \mathcal{I}} \bar{c}_{0}^{j}}
\end{aligned}
$$


(i): by the FOC, for all $(t, \sigma), \forall i \in \mathcal{I}, \bar{c}_{t-1}^{i}(\sigma)=\beta p^{i}\left(\sigma_{t-1} \mid \sigma^{t-2}\right) \bar{c}_{t-2}^{i}(\sigma) / q\left(\sigma_{t-1} \mid \sigma^{t-2}\right)$; so,

$$
\frac{\bar{c}_{t-1}^{i}(\sigma)}{\sum_{j \in \mathcal{I}} \bar{c}_{t-1}^{j}(\sigma)}=\frac{p^{i}\left(\sigma_{t-1} \mid \sigma^{t-2}\right) \bar{c}_{t-2}^{i}(\sigma)}{\sum_{j \in \mathcal{I}} p^{j}\left(\sigma_{t-1} \mid \sigma^{t-2}\right) \bar{c}_{t-2}^{j}(\sigma)}=\frac{p^{i}\left(\sigma_{t-1} \mid \sigma^{t-2}\right) \bar{c}_{t-2}^{i}(\sigma)}{p^{M}\left(\sigma_{t-1} \mid \sigma^{t-1}\right)} \frac{1}{\sum_{j \in \mathcal{I}} \bar{c}_{t-2}^{j}(\sigma)} .
$$

Proof of Lemma 2. From the FOC, for all $(t, \sigma)$,

$$
\forall i \in \mathcal{I}, \quad \bar{c}_{t}^{i}(\sigma) q\left(\sigma_{t} \mid \sigma^{t-1}\right)=\beta p^{i}\left(\sigma_{t} \mid \sigma^{t-1}\right) \bar{c}_{t-1}^{i}(\sigma)
$$

summing over $i$ and rearranging,

$$
\begin{aligned}
q\left(\sigma_{t} \mid \sigma^{t-1}\right) & =\sum_{i \in \mathcal{I}} \beta p^{i}\left(\sigma_{t} \mid \sigma^{t-1}\right) \frac{\bar{c}_{t-1}^{i}(\sigma)}{\sum_{j \in \mathcal{I}} \bar{c}_{t}^{i}(\sigma)} \\
& \Rightarrow p^{\mathrm{RN}}\left(\sigma_{t} \mid \sigma^{t-1}\right):=\frac{q\left(\sigma_{t} \mid \sigma^{t-1}\right)}{\sum_{\tilde{\sigma}_{t}} q\left(\tilde{\sigma}_{t} \mid \sigma^{t-1}\right)} \propto \sum_{i \in \mathcal{I}} p^{i}\left(\sigma_{t} \mid \sigma^{t-1}\right) \frac{\bar{c}_{t-1}^{i}(\sigma)}{\sum_{j \in \mathcal{I}} \bar{c}_{t}^{j}(\sigma)} .
\end{aligned}
$$

Proof of Lemma 3. In every equilibrium, for all $(t, \sigma)$,

$$
\begin{aligned}
p_{\gamma}^{\mathrm{RN}}\left(\sigma_{t} \mid \sigma^{t-1}\right) & :=\frac{q\left(\sigma_{t} \mid \sigma^{t-1}\right) \boldsymbol{e}_{t}(\sigma)^{\gamma}}{\sum_{\tilde{\sigma}_{t}} q\left(\tilde{\sigma}_{t} \mid \sigma^{t-1}\right) \boldsymbol{e}_{t}(\tilde{\sigma})^{\gamma}} \\
& \propto \sum_{i \in \mathcal{I}} p^{i}\left(\sigma_{t} \mid \sigma^{t-1}\right) \frac{\bar{c}_{t-1}^{i}(\sigma)}{\sum_{j \in \mathcal{I}} \bar{c}_{t}^{j}(\sigma)} \frac{\boldsymbol{e}_{t}(\sigma)^{\gamma}}{\boldsymbol{e}_{t-1}(\sigma)^{\gamma}} \\
& =\sum_{i \in \mathcal{I}} p^{i}\left(\sigma_{t} \mid \sigma^{t-1}\right) \frac{c_{t-1}^{i}(\sigma)^{\gamma}}{\sum_{j \in \mathcal{I}} c_{t}^{j}(\sigma)^{\gamma}} \frac{\left(\sum_{k \in \mathcal{I}} c_{t}^{k}(\sigma)\right)^{\gamma}}{\left(\sum_{t-1}^{l}(\sigma)\right)^{\gamma}} \\
& =\sum_{i \in \mathcal{I}} p^{i}\left(\sigma_{t} \mid \sigma^{t-1}\right) \frac{c_{t-1}^{i}(\sigma)^{\gamma}}{\left(\sum_{l \in \mathcal{I}} c_{t-1}^{l}(\sigma)\right)^{\gamma}} \frac{1}{\sum_{j \in \mathcal{I}} \frac{c_{t}^{j}(\sigma)^{\gamma}}{\left(\sum_{k \in \mathcal{I}}^{k}(\sigma)\right)^{\gamma}}} \\
& =\sum_{i \in \mathcal{I}} p^{i}\left(\sigma_{t} \mid \sigma^{t-1}\right) \frac{\phi_{t-1}^{i}(\sigma)^{\gamma}}{\sum_{j \in \mathcal{I}} \phi_{t}^{j}(\sigma)^{\gamma}}
\end{aligned}
$$

Lemma 4. Under A1, A2 (A5), and A4, if agents' beliefs are as in Definition 4 with $p^{C}=$ $p^{M}\left(p^{\mathrm{RN}}\right)$ and $\forall i \in \mathcal{I}, \alpha^{i} \in(0,1]$ then A3 is satisfied. 
Proof. By Definition 4, $p^{i}\left(\sigma_{t} \mid \sigma^{t-1}\right)=\left(1-\alpha^{i}\right) p^{C}\left(\sigma_{t} \mid \sigma^{t-1}\right)+\alpha^{i} \pi^{i}\left(\sigma_{t}\right)$ with both $\pi^{i}$ and $\alpha^{i}$ strictly positive $\forall i \in \mathcal{I}$. Therefore, for all $(t, \sigma), p^{i}\left(\sigma_{t} \mid \sigma^{t-1}\right) \geq \alpha^{i} \pi^{i}\left(\sigma_{t}\right)>0$.

Lemma 5. Under A1-A4, if agents' beliefs are as in Definition 4 with $p^{C}=p^{M}$, then

$$
\forall(t, \sigma), \forall j \in \mathcal{I} \cup M, \quad p^{j}\left(\sigma_{t} \mid \sigma^{t-1}\right) \in \operatorname{Conv}\left(\pi^{1}, \ldots, \pi^{I}\right) .
$$

Proof. Substituting $p^{i}\left(\sigma_{t} \mid \sigma^{t-1}\right)$ (Definition 4) in Definition 7,

$$
\forall(t, \sigma), \quad p^{M}\left(\sigma_{t} \mid \sigma^{t-1}\right)=\sum_{i \in \mathcal{I}}\left[\left(1-\alpha^{i}\right) p^{M}\left(\sigma_{t} \mid \sigma^{t-1}\right)+\alpha^{i} \pi^{i}\left(\sigma_{t}\right)\right] \frac{\bar{c}_{t-1}^{i}(\sigma)}{\sum_{j \in \mathcal{I}} \bar{c}_{t-1}^{j}(\sigma)} .
$$

Rearranging,

$$
\forall(t, \sigma), \quad p^{M}\left(\sigma_{t} \mid \sigma^{t-1}\right)=\sum_{i \in \mathcal{I}} \pi^{i}\left(\sigma_{t}\right) \frac{\alpha^{i} \bar{c}_{t-1}^{i}(\sigma)}{\sum_{j \in \mathcal{I}} \alpha^{j} \bar{c}_{t-1}^{j}(\sigma)} \in \operatorname{Conv}\left(\pi^{1}, \ldots, \pi^{I}\right) .
$$

So, $\forall i \in \mathcal{I}: \alpha^{i} \in(0,1), p^{i}\left(\sigma_{t} \mid \sigma^{t-1}\right) \in \operatorname{Conv}\left(\pi^{1}, \ldots, \pi^{I}\right)$ because it is the convex combination of two points in $\operatorname{Conv}\left(\pi^{1}, \ldots, \pi^{I}\right)$.

Proof of Proposition 2. (i) Let $\bar{\phi}_{0}^{i}:=\frac{\bar{c}_{0}^{i}}{\sum_{j \in \mathcal{I}} \bar{c}_{0}^{j}}$; for all $(t, \sigma)$,

$$
\begin{aligned}
p^{M}\left(\sigma^{t}\right) & ={ }^{\text {By Lem. } 1} \sum_{i \in \mathcal{I}} p^{i}\left(\sigma^{t}\right) \bar{\phi}_{0}^{i} \\
& \Rightarrow \forall i \in \mathcal{I}, \quad \ln p^{M}\left(\sigma^{t}\right) \geq \ln p^{i}\left(\sigma^{t}\right)+\ln \bar{\phi}_{0}^{i} \\
& \Rightarrow{ }^{(i)} \sum_{\tau=1}^{t} \ln p^{M}\left(\sigma_{\tau} \mid \sigma^{\tau-1}\right) \geq \sum_{\tau=1}^{t} \ln p^{i}\left(\sigma_{\tau} \mid \sigma^{\tau-1}\right)+\ln \bar{\phi}_{0}^{i} \\
& \Rightarrow \frac{1}{t} \sum_{\tau=1}^{t} \ln \frac{P\left(\sigma_{\tau}\right)}{p^{M}\left(\sigma_{\tau} \mid \sigma^{\tau-1}\right)} \leq \frac{1}{t} \sum_{\tau=1}^{t} \ln \frac{P\left(\sigma_{\tau}\right)}{p^{i}\left(\sigma_{\tau} \mid \sigma^{\tau-1}\right)}-\frac{1}{t} \ln \bar{\phi}_{0}^{i} \\
& \Rightarrow \lim _{t \rightarrow \infty}\left[\frac{1}{t}\left[\sum_{\tau=1}^{t} \ln \frac{P\left(\sigma_{\tau}\right)}{p^{M}\left(\sigma_{\tau} \mid \sigma^{\tau-1}\right)}-\sum_{\tau=1}^{t} d\left(P \| p_{\tau}^{M}\right)\right]+\frac{1}{t} \sum_{\tau=1}^{t} d\left(P \| p_{\tau}^{M}\right)\right] \\
& \leq \lim _{t \rightarrow \infty}\left[\frac{1}{t}\left[\sum_{\tau=1}^{t} \ln \frac{P\left(\sigma_{\tau}\right)}{p^{i}\left(\sigma_{\tau} \mid \sigma^{\tau-1}\right)}-\sum_{\tau=1}^{t} d\left(P \| p_{\tau}^{i}\right)\right]+\frac{1}{t} \sum_{\tau=1}^{t} d\left(P \| p_{\tau}^{i}\right)-\frac{1}{t} \ln \bar{\phi}_{0}^{i}\right] \\
& \Rightarrow{ }^{(i i)} \bar{d}\left(P \| p^{M}\right) \leq \bar{d}\left(P \| p^{i}\right), \quad P \text {-a.s., by the SLLNMD. }
\end{aligned}
$$

(i) Remembering that for $j=i, M$ and for all $(t, \sigma)$,

$$
p^{j}\left(\sigma^{t}\right):=\prod_{\tau=1}^{t} p^{j}\left(\sigma_{\tau} \mid \sigma^{\tau-1}\right) .
$$


(ii) It follows from the Strong Law of Large Number for Martingale Differences (SLLNMD) (see also Sandroni (2000)) that guarantees that for $j=i, M$,

$$
\lim _{t \rightarrow \infty} \frac{1}{t}\left[\sum_{\tau=1}^{t} \ln \frac{P\left(\sigma_{\tau}\right)}{p^{j}\left(\sigma_{\tau} \mid \sigma^{\tau-1}\right)}-\sum_{\tau=1}^{t} d\left(P \| p_{\tau}^{j}\right)\right]=0, \quad P \text {-a.s. }
$$

(ii): We proceed by proving the contrapositive statement: $\bar{d}\left(P \| p^{M}\right)<\bar{d}\left(P \| p^{i}\right)$, $P$-a.s. $\Rightarrow$ agent $i$ vanishes-the opposite inequality is ruled out by (i).

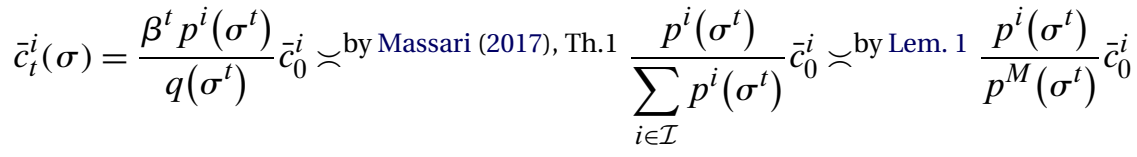

$$
\begin{aligned}
& \Rightarrow \lim _{t \rightarrow \infty} \frac{1}{t} \ln \bar{c}_{t}^{i}(\sigma)=\lim _{t \rightarrow \infty} \frac{1}{t} \ln \frac{p^{i}\left(\sigma^{t}\right)}{p^{M}\left(\sigma^{t}\right)}+\frac{1}{t} \ln \bar{c}_{0}^{i} \\
& =\lim _{t \rightarrow \infty} \frac{1}{t}\left[\ln \frac{P\left(\sigma^{t}\right)}{p^{M}\left(\sigma^{t}\right)}-\ln \frac{P\left(\sigma^{t}\right)}{p^{i}\left(\sigma^{t}\right)}\right] \\
& =\bar{d}\left(P \| p^{M}\right)-\bar{d}\left(P \| p^{i}\right), \quad P \text {-a.s., by the SLLNMD }
\end{aligned}
$$

Therefore,

$$
\begin{aligned}
\bar{d}\left(P \| p^{M}\right)<\bar{d}\left(P \| p^{i}\right), \quad P \text {-a.s. } & \Rightarrow \lim _{t \rightarrow \infty} \frac{1}{t} \ln \bar{c}_{t}^{i}(\sigma)<0, \quad P \text {-a.s. } \\
& \Rightarrow \ln \bar{c}_{t}^{i}(\sigma) \rightarrow-\infty, \quad P \text {-a.s. } \\
& \Rightarrow \frac{1}{u^{i \prime}\left(c_{t}^{i}(\sigma)\right)} \rightarrow 0, \quad P \text {-a.s. } \\
& \Rightarrow c_{t}^{i}(\sigma) \rightarrow 0, \quad P \text {-a.s. by A1 } \\
& \Rightarrow \text { agent } i \text { vanishes. }
\end{aligned}
$$

Proof of Proposition 3. $\forall(t, \sigma)$,

$$
\begin{aligned}
d\left(P \| p_{t}^{i}\right) & \left.=d\left(P \|\left(1-\alpha^{i}\right) p_{t}^{M}+\alpha^{i} \pi^{i}\right)\right) \\
& \leq{ }^{(i)}\left(1-\alpha^{i}\right) d\left(P \| p_{t}^{M}\right)+\alpha^{i} d\left(P \| \pi^{i}\right) ; \quad \text { by strict convexity of } d(P \| \cdot) \\
& \Rightarrow \bar{d}\left(P \| p^{i}\right) \leq\left(1-\alpha^{i}\right) \bar{d}\left(P \| p^{M}\right)+\alpha^{i} \bar{d}\left(P \| \pi^{i}\right) ; \quad \text { summing and averaging over } t \\
& \Rightarrow \bar{d}\left(P \| p^{i}\right) \leq \bar{d}\left(P \| \pi^{i}\right), \quad P \text {-a.s.; because } \bar{d}\left(P \| p^{M}\right) \leq \text { by Prop. } 2 \bar{d}\left(P \| p^{i}\right)
\end{aligned}
$$

Moreover, if there exists an $\epsilon>0$ such that $\left\|p_{t}^{M}-\pi^{i}\right\|>\epsilon$ a in positive fraction of periods, then $\bar{d}\left(P \| p^{i}\right)<\bar{d}\left(P \| \pi^{i}\right)$ because inequality (i) is strict in a positive fraction of periods by continuity and strict convexity of $d(P \| \cdot)$. 
Proof of Proposition 4. WOC $^{M} \Rightarrow$ beliefs must be diverse. We prove the contrapositive statement:

$$
\left.\pi^{\mathrm{BCP}}=\pi^{\mathrm{BIP}} \Rightarrow \bar{d}\left(P \| p^{M}\right) \geq \bar{d}\left(P \| \pi^{\mathrm{BIP}}\right), \quad P \text {-a.s. (i.e., no } \mathrm{WOC}^{M}\right) .
$$

$\forall(t, \sigma), p_{t}^{M} \in$ By Lem. $5^{C o n v}\left(\pi^{1}, \ldots, \pi^{I}\right)$ and $\pi^{\mathrm{BCP}}:=\operatorname{argmin}_{p \in \operatorname{Conv}\left(\pi^{1}, \ldots, \pi^{I}\right)} d(P \| p)$.

Thus, for every choice of $\alpha^{i} \in(0,1], \forall \sigma, \bar{d}\left(P \| p^{M}\right) \geq \bar{d}\left(P \| \pi^{\mathrm{BCP}}\right)={ }^{\mathrm{By}} H_{0} \bar{d}\left(P \| \pi^{\mathrm{BIP}}\right)$.

$\mathrm{WOC}^{M} \Rightarrow \exists i: \alpha^{i} \in(0,1)$. We prove the contrapositive statement:

$$
\left.\forall i \in \mathcal{I}, \quad \alpha^{i}=1 \Rightarrow \bar{d}\left(P \| p^{M}\right) \geq \bar{d}\left(P \| \pi^{\mathrm{BIP}}\right), \quad P \text {-a.s.(i.e., no } \mathrm{WOC}^{M}\right) .
$$

$\forall i \in \mathcal{I}, \alpha^{i}=1 \Rightarrow \forall i \in \mathcal{I}, p^{i}=\pi^{i}$, and the result follows from Proposition 2(i).

The following two lemmas are necessary for the proof of Proposition 6.

Lemma 6. Under A1-A5, if agents' utilities are CRRA and the aggregate endowment is constant, for all $(t, \sigma)$,

$$
\begin{aligned}
& \forall i, \quad \gamma^{i} \geq 1 \Rightarrow \frac{1}{\beta} \sum_{\sigma_{t}} q\left(\sigma_{t} \mid\right) \leq 1, \\
& \forall i, \quad \gamma^{i} \leq 1 \Rightarrow \frac{1}{\beta} \sum_{\sigma_{t}} q\left(\sigma_{t} \mid\right) \geq 1,
\end{aligned}
$$

with equality if and only if the consumption shares distribution is degenerate, or $\gamma^{i}=1$ for all agents, or all agents have identical beliefs.

Proof. On every equilibrium path $\forall(t, \sigma)$ and for all $i$,

$$
c_{t}^{i}(\sigma)=\left(\frac{\beta p^{i}\left(\sigma_{t} \mid\right)}{q\left(\sigma_{t} \mid\right)}\right)^{\frac{1}{\gamma^{i}}} c_{t-1}^{i}(\sigma) .
$$

Multiplying both sides of the equation by $q\left(\sigma_{t} \mid\right) / \beta$,

$$
\frac{q\left(\sigma_{t} \mid\right)}{\beta} c_{t}^{i}(\sigma)=p^{i}\left(\sigma_{t} \mid\right)^{\frac{1}{\gamma^{i}}}\left(\frac{q\left(\sigma_{t} \mid\right)}{\beta}\right)^{1-\frac{1}{\gamma^{i}}} c_{t-1}^{i}(\sigma) .
$$

Summing on both sides of the equation over all the agents,

$$
\frac{q\left(\sigma_{t} \mid\right)}{\beta} \sum_{i \in \mathcal{I}} c_{t}^{i}(\sigma)=\sum_{i \in \mathcal{I}} p^{i}\left(\sigma_{t} \mid\right)^{\frac{1}{\gamma^{i}}}\left(\frac{q\left(\sigma_{t} \mid\right)}{\beta}\right)^{1-\frac{1}{\gamma^{i}}} c_{t-1}^{i}(\sigma) .
$$

Dividing on both sides of the equation by the aggregate endowment (which is constant over $t$ )

$$
\frac{q\left(\sigma_{t} \mid\right)}{\beta}=\sum_{i \in \mathcal{I}} p^{i}\left(\sigma_{t} \mid\right)^{\frac{1}{\gamma^{i}}}\left(\frac{q\left(\sigma_{t} \mid\right)}{\beta}\right)^{1-\frac{1}{\gamma^{i}}} \phi_{t-1}^{i},
$$

where $\left[\phi_{t-1}^{1}, \ldots, \phi_{t-1}^{I}\right]$ is the consumption shares distribution in $\left(t-1, \sigma^{t-1}\right)$. 
Summing on both sides of the equation over the states:

$$
\sum_{\sigma_{t}} \frac{q\left(\sigma_{t} \mid\right)}{\beta}=\sum_{i \in \mathcal{I}} \sum_{\sigma_{t}} p^{i}\left(\sigma_{t} \mid\right)^{\frac{1}{\gamma^{i}}}\left(\frac{q\left(\sigma_{t} \mid\right)}{\beta}\right)^{1-\frac{1}{\gamma^{i}}} \phi_{t-1}^{i} .
$$

Multiplying the right-hand side by

$$
\frac{\prod_{k \in \mathcal{I}}\left(\sum_{\sigma_{t}} \frac{q\left(\sigma_{t} \mid\right)}{\beta}\right)^{1-\frac{1}{\gamma^{k}}}}{\prod_{j \in \mathcal{I}}\left(\sum_{\sigma_{t}} \frac{q\left(\sigma_{t} \mid\right)}{\beta}\right)^{1-\frac{1}{\gamma^{j}}}}=1
$$

we can express the left-hand side as a function of the risk-neutral probabilities:

$$
\sum_{\sigma_{t}} \frac{q\left(\sigma_{t} \mid\right)}{\beta}=\sum_{i \in \mathcal{I}} \sum_{\sigma_{t}} p^{i}\left(\sigma_{t} \mid\right)^{\frac{1}{\gamma^{i}}} p^{\mathrm{RN}}\left(\sigma_{t} \mid\right)^{1-\frac{1}{\gamma^{i}}} \phi_{t-1}^{i} \frac{\prod_{k \in \mathcal{I}}\left(\sum_{\sigma_{t}} \frac{q\left(\sigma_{t} \mid\right)}{\beta}\right)^{1-\frac{1}{\gamma^{k}}}}{\prod_{j \neq i}\left(\sum_{\sigma_{t}} \frac{q\left(\sigma_{t} \mid\right)}{\beta}\right)^{1-\frac{1}{\gamma^{j}}}} .
$$

- Let us focus on the case in which $\forall i, \gamma^{i} \geq 1$.

Let $i^{*}:=\operatorname{argmax}_{i \in \mathcal{I}}\left(\sum_{\sigma_{t}} q\left(\sigma_{t} \mid\right) / \beta\right)^{1-\frac{1}{\gamma^{i}}}$; so that

$$
\forall k, i \in \mathcal{I}, \quad \frac{\prod_{k \neq i^{*}}\left(\sum_{\sigma_{t}} \frac{q\left(\sigma_{t} \mid\right)}{\beta}\right)^{1-\frac{1}{\gamma^{k}}}}{\prod_{j \neq i}\left(\sum_{\sigma_{t}} \frac{q\left(\sigma_{t} \mid\right)}{\beta}\right)^{1-\frac{1}{\gamma^{j}}}} \leq 1 .
$$

It follows that

$$
\begin{aligned}
& \sum_{\sigma_{t}} \frac{q\left(\sigma_{t} \mid\right)}{\beta} \\
& =\sum_{i \in \mathcal{I}} \sum_{\sigma_{t}} p^{i}\left(\sigma_{t} \mid\right)^{\frac{1}{\gamma^{i}}} p^{\mathrm{RN}}\left(\sigma_{t} \mid\right)^{1-\frac{1}{\gamma^{i}}} \phi_{t-1}^{i}\left(\sum_{\sigma_{t}} \frac{q\left(\sigma_{t} \mid\right)}{\beta}\right)^{1-\frac{1}{\gamma^{i^{*}}}} \frac{\prod_{k \neq i^{*}}\left(\sum_{\sigma_{t}} \frac{q\left(\sigma_{t} \mid\right)}{\beta}\right)^{1-\frac{1}{\gamma^{i}}}}{\prod_{j \neq i}\left(\sum_{\sigma_{t}} \frac{q\left(\sigma_{t} \mid\right)}{\beta}\right)^{1-\frac{1}{\gamma^{j}}}} \\
& \leq \sum_{i \in \mathcal{I}} \sum_{\sigma_{t}} p^{i}\left(\sigma_{t} \mid\right)^{\frac{1}{\gamma^{i}}} p^{\mathrm{RN}}\left(\sigma_{t} \mid\right)^{1-\frac{1}{\gamma^{i}}} \phi_{t-1}^{i}\left(\sum_{\sigma_{t}} \frac{q\left(\sigma_{t} \mid\right)}{\beta}\right)^{1-\frac{1}{\gamma^{i^{*}}}} .
\end{aligned}
$$


Rearranging,

$$
\begin{aligned}
\left(\sum_{\sigma_{t}} \frac{q\left(\sigma_{t} \mid\right)}{\beta}\right)^{\frac{1}{\gamma^{i}}} & \leq \sum_{i \in \mathcal{I}} \sum_{\sigma_{t}} p^{i}\left(\sigma_{t} \mid\right)^{\frac{1}{\gamma^{i}}} p^{\mathrm{RN}}\left(\sigma_{t} \mid\right)^{1-\frac{1}{\gamma^{i}}} \phi_{t-1}^{i} \\
& \leq{ }^{(i)} \sum_{i \in \mathcal{I}} \sum_{\sigma_{t}}\left(\frac{1}{\gamma^{i}} p^{i}\left(\sigma_{t} \mid\right)+\left(1-\frac{1}{\gamma^{i}}\right) p^{\mathrm{RN}}\left(\sigma_{t} \mid\right)\right) \phi_{t-1}^{i}=1 \\
& \Rightarrow \sum_{\sigma_{t}} \frac{q\left(\sigma_{t} \mid\right)}{\beta} \leq 1 .
\end{aligned}
$$

(i): $\forall i \in \mathcal{I}, \gamma^{i} \geq 1 \Rightarrow \forall \sigma_{t}, p^{i}\left(\sigma_{t} \mid\right)^{\frac{1}{\gamma^{i}}} p^{\mathrm{RN}}\left(\sigma_{t} \mid\right)^{1-\frac{1}{\gamma^{i}}} \leq \frac{1}{\gamma^{i}} p^{i}\left(\sigma_{t} \mid\right)+\left(1-\frac{1}{\gamma^{i}}\right) p^{\mathrm{RN}}\left(\sigma_{t} \mid\right)$, by the ordering of the arithmetic and geometric means.

- Let us focus on the case in which $\forall i, \gamma^{i} \leq 1$.

Let $i^{* *}:=\operatorname{argmin}_{i \in \mathcal{I}}\left(\sum_{\sigma_{t}} q\left(\sigma_{t} \mid\right) / \beta\right)^{1-\frac{1}{\gamma^{i}}}$; so that

$$
\forall k, i \in \mathcal{I}, \quad \frac{\prod_{k \neq i^{* *}}\left(\sum_{\sigma_{t}} \frac{q\left(\sigma_{t} \mid\right)}{\beta}\right)^{1-\frac{1}{\gamma^{k}}}}{\prod_{j \neq i}\left(\sum_{\sigma_{t}} \frac{q\left(\sigma_{t} \mid\right)}{\beta}\right)^{1-\frac{1}{\gamma^{j}}}} \geq 1 .
$$

Proceeding as above, we obtain the opposite inequality:

$$
\left(\sum_{\sigma_{t}} \frac{q\left(\sigma_{t} \mid\right)}{\beta}\right)^{\frac{1}{\gamma^{i *}}} \geq \sum_{i \in \mathcal{I}} \sum_{\sigma_{t}} p^{i}\left(\sigma_{t} \mid\right)^{\frac{1}{\gamma^{i}}} p^{\mathrm{RN}}\left(\sigma_{t} \mid\right)^{1-\frac{1}{\gamma^{i}}} \phi_{t-1}^{i} .
$$

The result follows by showing that

$$
\gamma^{i} \leq 1 \quad \forall i \Rightarrow \ln \sum_{i \in \mathcal{I}} \sum_{\sigma_{t}} p^{i}\left(\sigma_{t} \mid\right)^{\frac{1}{\gamma^{i}}} p^{\mathrm{RN}}\left(\sigma_{t} \mid\right)^{1-\frac{1}{\gamma^{i}}} \phi_{t-1}^{i} \geq 0 .
$$

For convenience, let $\forall i, \eta_{i}:=1 / \gamma^{i}$; so that $\forall i, \eta_{i} \in(1, \infty)$.

$$
\begin{aligned}
& \ln \sum_{i \in \mathcal{I}} \sum_{\sigma_{t}} p^{i}\left(\sigma_{t} \mid\right)^{\frac{1}{\gamma^{i}}} p^{\mathrm{RN}}\left(\sigma_{t} \mid\right)^{1-\frac{1}{\gamma^{i}}} \phi_{t-1}^{i} \\
& \quad=\ln \sum_{i \in \mathcal{I}} \sum_{\sigma_{t}} \frac{p^{i}\left(\sigma_{t} \mid\right)^{\eta_{i}}}{p^{\mathrm{RN}}\left(\sigma_{t} \mid\right)^{\eta_{i}-1}} \phi_{t-1}^{i} \\
& \quad \geq{ }^{(i)} \sum_{i \in \mathcal{I}} \phi_{t-1}^{i} \ln \sum_{\sigma_{t}} \frac{p^{i}\left(\sigma_{t} \mid\right)^{\eta_{i}}}{p^{\mathrm{RN}}\left(\sigma_{t} \mid\right)^{\eta_{i}-1}} \\
& \quad=\sum_{i \in \mathcal{I}}\left(\eta_{i}-1\right) \phi_{t-1}^{i}\left(\frac{1}{\eta_{i}-1} \ln \sum_{\sigma_{t}} \frac{p^{i}\left(\sigma_{t} \mid\right)^{\eta_{i}}}{p^{\mathrm{RN}}\left(\sigma_{t} \mid\right)^{\eta_{i}-1}}\right)
\end{aligned}
$$




$$
\begin{aligned}
& { }^{(i i)} \sum_{i \in \mathcal{I}}\left(\eta_{i}-1\right) \phi_{t-1}^{i} D_{\eta^{i}}\left(p_{t}^{i} \| p_{t}^{\mathrm{RN}}\right) \\
& \geq^{(i i i)} 0 .
\end{aligned}
$$

(i) By concavity of log.

(ii) Recognizing the definition of the Rényi divergence $\left(D_{\eta^{i}}\left(p_{t}^{i} \| p_{t}^{\mathrm{RN}}\right)\right)$ between $p_{t}^{i}$ and $p_{t}^{\mathrm{RN}}$ (Rényi 1961, Van Erven and Harremos 2014).

(iii) Rény divergence is weakly positive, it equals 0 iff $p^{i}=p^{\mathrm{RN}}$ (Van Erven and Harremos 2014).

An inspection of Equation (6) shows that equality holds if and only if

- either the consumption share distribution is degenerate because

$$
\phi_{t-1}^{i}=1 \Rightarrow p_{t}^{i}=p_{t}=p_{t}^{\mathrm{RN}} \Rightarrow\left(\sum_{\sigma_{t}} \frac{q\left(\sigma_{t} \mid\right)}{\beta}\right)^{\frac{1}{\gamma^{i}}}=\sum_{i \in \mathcal{I}} \sum_{\sigma_{t}} p_{t}\left(\sigma_{t} \mid\right)^{\frac{1}{\gamma^{i}}} p_{t}\left(\sigma_{t} \mid\right)^{1-\frac{1}{\gamma^{i}}} \phi_{t-1}^{i}=1 ;
$$

- or $\gamma^{i}=1$ for all agents because $\sum_{\sigma_{t}} q\left(\sigma_{t} \mid\right) / \beta=\sum_{i \in \mathcal{I}} \sum_{\sigma_{t}} p^{i}\left(\sigma_{t} \mid\right)=1$;

- or all agents have identical beliefs because

$$
\forall i, \quad p_{t}^{i}=p_{t}=p_{t}^{\mathrm{RN}} \Rightarrow \forall i, \quad\left(\sum_{\sigma_{t}} \frac{q\left(\sigma_{t} \mid\right)}{\beta}\right)^{\frac{1}{\gamma^{i}}}=\sum_{i \in \mathcal{I}} \sum_{\sigma_{t}} p_{t}\left(\sigma_{t} \mid\right)^{\frac{1}{\gamma^{i}}} p_{t}\left(\sigma_{t} \mid\right)^{1-\frac{1}{\gamma^{i}}} \phi_{t-1}^{i}=1
$$

Lemma 7. Under A1-A5, if all agents have identical CRRA utility then, for all $(t, \sigma)$ :

$$
\begin{aligned}
& \forall i, \quad \gamma^{i} \geq 1 \Rightarrow \frac{1}{\beta} \sum_{\sigma_{t}} q\left(\sigma_{t} \mid \sigma^{t-1}\right)\left(\frac{\boldsymbol{e}_{t}(\sigma)}{\boldsymbol{e}_{t-1}(\sigma)}\right)^{\gamma} \leq 1, \\
& \forall i, \quad \gamma^{i} \leq 1 \Rightarrow \frac{1}{\beta} \sum_{\sigma_{t}} q\left(\sigma_{t} \mid \sigma^{t-1}\right)\left(\frac{\boldsymbol{e}_{t}(\sigma)}{\boldsymbol{e}_{t-1}(\sigma)}\right)^{\gamma} \geq 1 ;
\end{aligned}
$$

with equality if and only if the consumption shares distribution is degenerate, or $\gamma^{i}=1$ for all agents, or all agents have identical beliefs.

Proof. This proof mimics that of Lemma 6. On every equilibrium path $\forall(t, \sigma)$ and for all $i$,

$$
c_{t}^{i}(\sigma)=\left(\frac{\beta p^{i}\left(\sigma_{t} \mid\right)}{q\left(\sigma_{t} \mid\right)}\right)^{\frac{1}{\gamma}} c_{t-1}^{i}(\sigma) .
$$

Multiplying both sides by $\left(q\left(\sigma_{t} \mid\right) / \beta\right)\left(\boldsymbol{e}_{t}(\sigma) / \boldsymbol{e}_{t-1}(\sigma)\right)^{\gamma-1}$, we have

$$
\frac{q\left(\sigma_{t} \mid\right)}{\beta}\left(\frac{\boldsymbol{e}_{t}(\sigma)}{\boldsymbol{e}_{t-1}(\sigma)}\right)^{\gamma-1} c_{t}^{i}(\sigma)=p^{i}\left(\sigma_{t} \mid\right)^{\frac{1}{\gamma}}\left(\frac{q\left(\sigma_{t} \mid\right)}{\beta}\left(\frac{\boldsymbol{e}_{t}(\sigma)}{\boldsymbol{e}_{t-1}(\sigma)}\right)^{\gamma}\right)^{1-\frac{1}{\gamma}} c_{t-1}^{i}(\sigma) .
$$


Summing on both sides of the equation over agents, $i$,

$$
\frac{q\left(\sigma_{t} \mid\right)}{\beta}\left(\frac{\boldsymbol{e}_{t}(\sigma)}{\boldsymbol{e}_{t-1}(\sigma)}\right)^{\gamma-1} \sum_{i \in \mathcal{I}} c_{t}^{i}(\sigma)=\sum_{i \in \mathcal{I}} p^{i}\left(\sigma_{t} \mid\right)^{\frac{1}{\gamma}}\left(\frac{q\left(\sigma_{t} \mid\right)}{\beta}\left(\frac{\boldsymbol{e}_{t}(\sigma)}{\boldsymbol{e}_{t-1}(\sigma)}\right)^{\gamma}\right)^{1-\frac{1}{\gamma}} c_{t-1}^{i}(\sigma) .
$$

Noticing that $\boldsymbol{e}_{t}(\sigma)=\sum_{i \in \mathcal{I}} c_{t}^{i}(\sigma)$ and $\boldsymbol{e}_{t-1}(\sigma)=\sum_{i \in \mathcal{I}} c_{t-1}^{i}(\sigma)$, simplifying and rearranging

$$
\frac{q\left(\sigma_{t} \mid\right)}{\beta}\left(\frac{\boldsymbol{e}_{t}(\sigma)}{\boldsymbol{e}_{t-1}(\sigma)}\right)^{\gamma}=\sum_{i \in \mathcal{I}} p^{i}\left(\sigma_{t} \mid\right)^{\frac{1}{\gamma}}\left(\frac{q\left(\sigma_{t} \mid\right)}{\beta}\left(\frac{\boldsymbol{e}_{t}(\sigma)}{\boldsymbol{e}_{t-1}(\sigma)}\right)^{\gamma}\right)^{1-\frac{1}{\gamma}} \phi_{t-1}^{i}(\sigma),
$$

where $\left[\phi_{t-1}^{1}, \ldots, \phi_{t-1}^{I}\right]$ is the consumption shares distribution in $\left(t-1, \sigma^{t-1}\right)$.

Summing on both sides of the equation over states:

$$
\sum_{\sigma_{t}} \frac{q\left(\sigma_{t} \mid\right)}{\beta}\left(\frac{\boldsymbol{e}_{t}(\sigma)}{\boldsymbol{e}_{t-1}(\sigma)}\right)^{\gamma}=\sum_{i \in \mathcal{I}} \sum_{\sigma_{t}} p^{i}\left(\sigma_{t} \mid\right)^{\frac{1}{\gamma}}\left(\frac{q\left(\sigma_{t} \mid\right)}{\beta}\left(\frac{\boldsymbol{e}_{t}(\sigma)}{\boldsymbol{e}_{t-1}(\sigma)}\right)^{\gamma}\right)^{1-\frac{1}{\gamma}} \phi_{t-1}^{i}(\sigma) .
$$

Dividing both sides by $\left(\sum_{\sigma_{t}} \frac{q\left(\sigma_{t}\right)\left(\frac{e_{t}(\sigma)}{e_{t-1}(\sigma)}\right)^{\gamma}}{\beta}\right)^{1-\frac{1}{\gamma}}$, we obtain

$$
\left[\sum_{\sigma_{t}} \frac{q\left(\sigma_{t} \mid\right)\left(\frac{\boldsymbol{e}_{t}(\sigma)}{\boldsymbol{e}_{t-1}(\sigma)}\right)^{\gamma}}{\beta}\right]^{\frac{1}{\gamma}}=\sum_{i \in \mathcal{I}} \sum_{\sigma_{t}} p^{i}\left(\sigma_{t} \mid\right)^{\frac{1}{\gamma}} p^{\mathrm{RN}}\left(\sigma_{t} \mid\right)^{1-\frac{1}{\gamma}} \phi_{t-1}^{i} .
$$

The rest of the proof is now identical to that of Lemma 6, substituting Equation (10) into Equations (7) and (8) to study the cases $\gamma \geq 1, \gamma \leq 1$, respectively.

Proof of Proposition 6. Let us start from the case of constant aggregate endowment.

$$
\text { Note that } \forall(t, \sigma), \quad \begin{aligned}
\ln p^{\mathrm{RN}}\left(\sigma^{t}\right) & =\ln \prod_{\tau=1}^{t} p^{\mathrm{RN}}\left(\sigma_{\tau} \mid\right)=\ln \prod_{\tau=1}^{t} \frac{q\left(\sigma_{\tau} \mid\right)}{\sum_{\hat{\sigma}_{\tau}} q\left(\hat{\sigma}_{\tau} \mid\right)} \\
& =\ln \frac{q\left(\sigma^{t}\right)}{\beta^{t}}-\sum_{\tau=1}^{t} \ln \left(\frac{1}{\beta} \sum_{\sigma_{\tau}} q\left(\sigma_{\tau} \mid\right)\right)
\end{aligned}
$$

by Massari (2017), Theorem $1 \asymp \ln \left(\sum_{i \in \mathcal{I}} p^{i}\left(\sigma^{t}\right)\right)-\sum_{\tau=1}^{t} \ln \left(\frac{1}{\beta} \sum_{\sigma_{\tau}} q\left(\sigma_{\tau} \mid\right)\right)$.

Therefore,

$$
\begin{aligned}
& \bar{d}\left(P \| p^{M}\right)-\bar{d}\left(P \| p^{\mathrm{RN}}\right) \\
& \quad=\lim _{t \rightarrow \infty} \frac{1}{t}\left(\ln p^{\mathrm{RN}}\left(\sigma^{t}\right)-\ln p^{M}\left(\sigma^{t}\right)\right), \quad P \text {-a.s., by the SLLNMD }
\end{aligned}
$$




$$
\begin{aligned}
& =\lim _{t \rightarrow \infty} \frac{1}{t}\left(\ln \sum_{i \in \mathcal{I}} p^{i}\left(\sigma^{t}\right)-\frac{1}{t} \sum_{\tau=1}^{t} \ln \left(\frac{1}{\beta} \sum_{\sigma_{\tau}} q\left(\sigma_{\tau} \mid\right)\right)-\ln p^{M}\left(\sigma^{t}\right)\right) \\
& ={ }^{\text {By Lem. } 1} \lim _{t \rightarrow \infty} \frac{1}{t}\left(\ln \sum_{i \in \mathcal{I}} p^{i}\left(\sigma^{t}\right)-\frac{1}{t} \sum_{\tau=1}^{t} \ln \left(\frac{1}{\beta} \sum_{\sigma_{\tau}} q\left(\sigma_{\tau} \mid\right)\right)-\ln \sum_{i \in \mathcal{I}} p^{i}\left(\sigma^{t}\right)\right) \\
& =-\lim _{t \rightarrow \infty} \frac{1}{t} \sum_{\tau=1}^{t} \ln \left(\frac{1}{\beta} \sum_{\sigma_{\tau}} q\left(\sigma_{\tau} \mid\right)\right) \\
& \text { and Lemma } 6 \Rightarrow \begin{cases}\geq 0 & \text { if } \forall i, \gamma^{i} \in[1, \infty), \\
\leq 0 & \text { if } \forall i, \gamma^{i} \in(0,1] ;\end{cases}
\end{aligned}
$$

where inequalities are strict if and only if there is long-run heterogeneity a positive fraction of periods (i.e., if and only if at least one surviving agent has $\alpha \in(0,1))$ and not all the surviving agents have log utility (by Lemma 6).

- The proof of the case of common CRRA utility and aggregate risk is obtained by repeating the same steps but replacing $\left(\sum_{\sigma_{t}} q\left(\sigma_{t} \mid\right) / \beta\right)$ and Lemma 6 with $\left(\sum_{\sigma_{t}} q\left(\sigma_{t} \mid\right) / \beta\left(\boldsymbol{e}_{t}(\sigma) / \boldsymbol{e}_{t-1}(\sigma)\right)^{\gamma}\right)$ and Lemma 7 , respectively.

Proof of Proposition 7. The condition on $p^{i}$ is sufficient to guarantee that agent BIP does not dominate-otherwise, agent $i$ would be more accurate than agent BIP, violating Proposition 1. With long-run heterogeneity, $\bar{d}\left(P \| p^{\mathrm{RN}}\right)<{ }^{\text {Prop. } 6(i i i)} \bar{d}\left(P \| p^{M}\right)$ and the result follows because

$$
\bar{d}\left(P \| p^{\mathrm{RN}}\right)<\text { Prop. 6(iii) } \bar{d}\left(P \| p^{M}\right) \leq \text { Prop. } 2 \bar{d}\left(P \| p^{\mathrm{BIP}}\right) \leq \bar{d}\left(P \| \pi^{\mathrm{BIP}}\right),
$$

where the last inequality follows from Proposition 3, if agent BIP mixes with $p^{M}$, and from the following argument if agent BIP mixes with $p^{\mathrm{RN}}$. For all $(t, \sigma)$,

$$
\begin{aligned}
& d\left(P \| p_{t}^{\mathrm{BIP}}\right)\left.=d\left(P \|\left(1-\alpha^{\mathrm{BIP}}\right) p_{t}^{\mathrm{RN}}+\alpha^{\mathrm{BIP}} \pi^{\mathrm{BIP}}\right)\right) \\
& \leq\left(1-\alpha^{\mathrm{BIP}}\right) d\left(P \| p_{t}^{\mathrm{RN}}\right)+\alpha^{\mathrm{BIP}} d\left(P \| \pi^{\mathrm{BIP}}\right) ; \quad \text { by strict convexity of } d(P \| \cdot) \\
& \Rightarrow \bar{d}\left(P \| p^{\mathrm{BIP}}\right) \leq\left(1-\alpha^{\mathrm{BIP}}\right) \bar{d}\left(P \| p^{\mathrm{RN}}\right)+\alpha^{\mathrm{BIP}} \bar{d}\left(P \| \pi^{\mathrm{BIP}}\right) ;
\end{aligned}
$$

summing and averaging over $t$

$\Rightarrow \bar{d}\left(P \| p^{\text {BIP }}\right)<{ }^{\text {Prop. 6(iii) }}\left(1-\alpha^{\mathrm{BIP}}\right) \bar{d}\left(P \| p^{M}\right)+\alpha^{\mathrm{BIP}} \bar{d}\left(P \| \pi^{\mathrm{BIP}}\right)$;

by long-run heterogeneity

$$
\begin{aligned}
& \leq{ }^{\text {Prop. } 2}\left(1-\alpha^{\mathrm{BIP}}\right) \bar{d}\left(P \| p^{\mathrm{BIP}}\right)+\alpha^{\mathrm{BIP}} \bar{d}\left(P \| \pi^{\mathrm{BIP}}\right) \\
\Rightarrow \bar{d}\left(P \| p^{\mathrm{BIP}}\right)<\bar{d}\left(P \| \pi^{\mathrm{BIP}}\right) . &
\end{aligned}
$$

Appendix B: Theorems 1 AND 2

The proof of Theorem 1 relies on Lemma 8. 
Lemma 8. Under A1-A4, if $\exists \hat{\mathcal{I}} \subset \mathcal{I}: P \in \operatorname{Conv}_{i \in \hat{\mathcal{I}}}\left(\pi^{i}\right), \forall i \in \hat{\mathcal{I}}, \alpha^{i} \in(0, \bar{\alpha}]$, and all agents in $\hat{\mathcal{I}}$ use $p^{M}$ for consensus; then $\exists \boldsymbol{\gamma} \in \Delta^{|\hat{\mathcal{I}}|}$ such that

$$
\bar{\alpha} \sum_{i \in \hat{\mathcal{I}}} \frac{\gamma^{i}}{\alpha^{i}} \bar{d}\left(P \| p^{M}\right)-\bar{\alpha} \sum_{i \in \hat{\mathcal{I}}} \frac{\gamma^{i}}{\alpha^{i}} \bar{d}\left(P \| p^{i}\right)=\lim _{t \rightarrow \infty} \frac{\bar{\alpha}}{t} \sum_{\tau=1}^{t} \mathrm{E}_{P}\left[\frac{P}{p_{t}^{M}}-1\right]-\left|O(\bar{\alpha})^{2}\right|,
$$

Proof. By assumption, for all $i \in \hat{\mathcal{I}}$, for all $(t, \sigma), p^{i}\left(\sigma_{t} \mid\right)=p^{M}\left(\sigma_{t} \mid\right)+\alpha^{i}\left(\pi^{i}\left(\sigma_{t}\right)-\right.$ $\left.p^{M}\left(\sigma_{t} \mid\right)\right)$.

We start by using Taylor's theorem to approximate

$$
\mathrm{E}_{P}\left[\ln \frac{p_{t}^{i}}{p_{t}^{M}}\right]=\mathrm{E}_{P}\left[\ln \left(1+\alpha^{i}\left(\frac{\pi^{i}\left(\sigma_{t}\right)}{p^{M}\left(\sigma_{t} \mid\right)}-1\right)\right)\right] \text { around } 1 .
$$

By Taylor's theorem with Lagrange's remainder, for some $\xi \in(0, x)$,

$$
\ln (1+x)=\ln 1+\left.\frac{1}{1+x}\right|_{x=0} x-\frac{1}{2(1+\xi)^{2}} x^{2} .
$$

For the purposes of our approximation, let $x^{M}\left(\alpha^{i}\right):=\alpha^{i}\left(\frac{\pi^{i}\left(\sigma_{t}\right)}{p^{M}\left(\sigma_{t} \mid\right)}-1\right)$.

Note that for all $i \in \hat{\mathcal{I}}$ :

(i) $\left(\frac{\pi^{i}\left(\sigma_{t}\right)}{p^{M}\left(\sigma_{t} \mid\right)}-1\right) \geq-1$ uniformly in $(t, \sigma)$ independently of the $\alpha^{i}$.

(ii) $\left(\frac{\pi^{i}\left(\sigma_{t}\right)}{p^{M}\left(\sigma_{t} \mid\right)}-1\right)$ is bounded above uniformly in $(t, \sigma)$, independently of the $\alpha^{i}$ :

$\exists K<\infty: \quad \forall(t, \sigma), \forall i \in \hat{\mathcal{I}},\left(\frac{\pi^{i}\left(\sigma_{t}\right)}{p^{M}\left(\sigma_{t} \mid\right)}-1\right)<K$.

Proof of (ii) Let for all $i \in \mathcal{I}$ and for all $(t, \sigma), \bar{\phi}_{t}^{i}(\sigma):=\frac{\bar{c}_{t}^{i}(\sigma)}{\sum_{j \in \mathcal{I}} \bar{c}_{t}^{j}(\sigma)}$; then, for all $(t, \sigma)$,

$$
\begin{aligned}
& p^{M}\left(\sigma_{t} \mid\right)={ }^{\text {By Def. } 2} \sum_{i \in \hat{\mathcal{I}}}\left(\left(1-\alpha^{i}\right) p^{M}\left(\sigma_{t} \mid\right)+\alpha^{i} \pi^{i}\left(\sigma_{t}\right)\right) \bar{\phi}_{t-1}^{i}(\sigma)+\sum_{j \in \mathcal{I} \backslash \hat{\mathcal{I}}} p^{j}\left(\sigma_{t} \mid\right) \bar{\phi}_{t-1}^{j}(\sigma) \\
& \Rightarrow \forall(t, \sigma), \\
& p^{M}\left(\sigma_{t} \mid\right)=\frac{\sum_{i \in \hat{\mathcal{I}}} \alpha^{i} \pi^{i}\left(\sigma_{t}\right) \bar{\phi}_{t-1}^{i}(\sigma)+\sum_{j \in \mathcal{I} \mid \hat{\mathcal{I}}} p^{j}\left(\sigma_{t} \mid\right) \bar{\phi}_{t-1}^{j}(\sigma)}{\sum_{i \in \hat{\mathcal{I}}} \alpha^{i} \bar{\phi}_{t-1}^{i}(\sigma)+\sum_{j \in \mathcal{I} \mid \hat{\mathcal{I}}} \bar{\phi}_{t-1}^{j}(\sigma)} \in \operatorname{Conv}_{i \in \hat{\mathcal{I}}, j \in \mathcal{I} \backslash \hat{\mathcal{I}}}\left(\pi^{i}, p^{j}\right) . \\
& \Rightarrow \forall(t, \sigma), \quad p^{M}\left(\sigma_{t} \mid\right) \geq \min _{i \in \hat{\mathcal{I}}, j \in \mathcal{I} \backslash \hat{\mathcal{I}}}\left(\pi^{i}, p^{j}\right)
\end{aligned}
$$


Definition 4 (for $i \in \hat{\mathcal{I}}$ ) and $\mathbf{A 3}$ (for $j \in \mathcal{I} \backslash \hat{\mathcal{I}}$ ) ensure that $\exists \epsilon>0$, independent of the $\alpha^{i}$ s of agents in $\hat{\mathcal{I}}: \min _{i \in \hat{\mathcal{I}}, j \in \mathcal{I} \backslash \hat{\mathcal{I}}}\left(\pi^{i}, p^{j}\right)>\epsilon$. So,

$$
\text { for all }(t, \sigma) \text { and for all } i \in \hat{\mathcal{I}}, \quad \frac{\pi^{i}}{p^{M}\left(\sigma_{t} \mid\right)}-1<\frac{\max _{i \in \hat{\mathcal{I}}} \pi^{i}}{\epsilon}=K<\infty .
$$

Taylor's theorem and the uniform bounds (i) and (ii) guarantee that for all $(t, \sigma)$ and for all $i \in \hat{\mathcal{I}}$,

$$
\begin{aligned}
-\frac{1}{2\left(1-\alpha^{i}\right)^{2}} \alpha^{i^{2}}\left(\frac{\pi^{i}\left(\sigma_{t}\right)}{p^{M}\left(\sigma_{t} \mid\right)}-1\right)^{2} & \leq \ln \left(1+x\left(\alpha^{i}\right)\right)-\alpha^{i}\left(\frac{\pi^{i}\left(\sigma_{t}\right)}{p^{M}\left(\sigma_{t} \mid\right)}-1\right) \\
& \leq-\frac{1}{2\left(1+\alpha^{i} K\right)^{2}} \alpha^{i^{2}}\left(\frac{\pi^{i}\left(\sigma_{t}\right)}{p^{M}\left(\sigma_{t} \mid\right)}-1\right)^{2},
\end{aligned}
$$

which justifies the equality

$$
\forall(t, \sigma), \forall i \in \hat{\mathcal{I}}, \quad \ln \left(1+x\left(\alpha^{i}\right)\right)=\alpha^{i}\left(\frac{\pi^{i}\left(\sigma_{t}\right)}{p^{M}\left(\sigma_{t} \mid\right)}-1\right)-\left|O\left(\left(\alpha^{i}\right)^{2}\right)\right| .
$$

Taking expectations,

$$
\mathrm{E}_{P}\left[\ln \frac{p_{t}^{i}}{p_{t}^{M}}\right]=\mathrm{E}_{P}\left[\ln \left(1+\alpha^{i}\left(\frac{\pi^{i}}{p_{t}^{M}}-1\right)\right)\right]=\alpha^{i} \mathrm{E}_{P}\left[\frac{\pi^{i}}{p_{t}^{M}}-1\right]-\left|O\left(\left(\alpha^{i}\right)^{2}\right)\right| .
$$

So that

$$
\begin{aligned}
\bar{d}\left(P \| p^{M}\right)-\bar{d}\left(P \| p^{i}\right) & =\lim _{t \rightarrow \infty} \frac{1}{t} \sum_{\tau=1}^{t} \mathrm{E}_{P}\left[\ln \frac{p_{\tau}^{i}}{p_{\tau}^{M}}\right] \\
& =\lim _{t \rightarrow \infty} \frac{\alpha^{i}}{t} \sum_{\tau=1}^{t} \mathrm{E}_{P}\left[\frac{\pi^{i}}{p_{\tau}^{M}}-1\right]-\left|O\left(\left(\alpha^{i}\right)^{2}\right)\right| .
\end{aligned}
$$

Let $\bar{\alpha}=\max _{i \in \hat{\mathcal{I}}^{\prime}}\left\{\alpha^{i}\right\}<1$, let $\gamma=\left[\gamma^{1}, \ldots, \gamma^{I}\right] \in \Delta^{|\hat{\mathcal{I}}|}$ be such that $\forall \sigma_{t} \in \mathcal{S}$, $\sum_{i \in \hat{\mathcal{I}}} \gamma^{i} \pi^{i}\left(\sigma_{t}\right)=P\left(\sigma_{t}\right)$-this vector exists because we assumed $P \in \operatorname{Conv}_{i \in \hat{\mathcal{I}}}\left(\pi^{i}\right)$-and let $\gamma_{\alpha}=\left[\gamma^{1} \bar{\alpha} / \alpha^{1}, \ldots, \gamma^{I} \bar{\alpha} / \alpha^{I}\right]$.

Equation (11) holds for all agents in $\hat{\mathcal{I}}$, therefore, it holds for their $\gamma_{\alpha}$ weighted sum:

$$
\begin{aligned}
\sum_{i \in \hat{\mathcal{I}}} \frac{\gamma^{i} \bar{\alpha}}{\alpha^{i}} \bar{d}\left(P \| p^{M}\right)-\sum_{i \in \hat{\mathcal{I}}} \frac{\gamma^{i} \bar{\alpha}}{\alpha^{i}} \bar{d}\left(P \| p^{i}\right) & =\sum_{i \in \hat{\mathcal{I}}} \frac{\gamma^{i} \bar{\alpha}}{\alpha^{i}}\left(\lim _{t \rightarrow \infty} \alpha^{i} \frac{1}{t} \sum_{\tau=1}^{t} \mathrm{E}_{P}\left[\frac{\pi^{i}}{p_{\tau}^{M}}-1\right]-\left|O\left(\left(\alpha^{i}\right)^{2}\right)\right|\right) \\
& =\lim _{t \rightarrow \infty} \frac{1}{t} \sum_{\tau=1}^{t} \bar{\alpha} \sum_{\gamma^{i}} \mathrm{E}_{P}\left[\gamma^{i} \frac{\pi^{i}}{p_{t}^{M}}-\gamma^{i}\right]-\sum_{i \in \hat{\mathcal{I}}} \frac{\gamma^{i} \bar{\alpha}}{\alpha^{i}}\left|O\left(\left(\alpha^{i}\right)^{2}\right)\right| \\
& =\lim _{t \rightarrow \infty} \frac{\bar{\alpha}}{t} \sum_{\tau=1}^{t} \mathrm{E}_{P}\left[\frac{P}{p_{t}^{M}}-1\right]-\left|O\left(\bar{\alpha}^{2}\right)\right| .
\end{aligned}
$$


Proof of Theorem 1. In equilibrium, the following inequalities must hold $P$-a.s.:

$$
\begin{aligned}
\forall i & \in \hat{\mathcal{I}}, \quad \bar{d}\left(P \| p^{M}\right)-\bar{d}\left(P \| p^{i}\right) \leq^{\text {By Prop. 2(i) } 0} \\
& \Rightarrow \forall \gamma \in \Delta^{|\hat{\mathcal{I}}|}, \quad \bar{\alpha} \sum_{i \in \hat{\mathcal{I}}} \frac{\gamma^{i}}{\alpha^{i}} \bar{d}\left(P \| p^{M}\right)-\bar{\alpha} \sum_{i \in \hat{\mathcal{I}}} \frac{\gamma^{i}}{\alpha^{i}} \bar{d}\left(P \| p^{i}\right) \leq 0 \\
& \Rightarrow{ }^{\text {By Lem. 8(i) }} \lim _{t \rightarrow \infty} \frac{\bar{\alpha}}{t} \sum_{\tau=1}^{t} \mathrm{E}_{P}\left[\frac{P}{p_{t}^{M}}-1\right] \leq\left|O(\bar{\alpha})^{2}\right| \\
& \Rightarrow \bar{d}\left(P \| p_{\bar{\alpha}}^{M}\right) \rightarrow^{\bar{\alpha} \rightarrow 0} 0
\end{aligned}
$$

The last implication holds because $p^{M}=P$ is the only minimizer for both the continuous nonnegative functions $d\left(P \| p_{t}^{M}\right)$ and $\mathrm{E}_{P}\left[P / p_{t}^{M}-1\right]$.

The proof of Theorem 2 is similar to that of Theorem 1 above, with Lemma 9 below replacing Lemma 8. For the sake of clarity ancillary results, needed to prove Lemma 9, Lemma 10, and Lemma 11, are presented after the proof of Theorem 2 at the end of this section.

LEMMA 9. Under A1-A5, if all agents in $\overline{\mathcal{I}}$ have CRRA utility with $\gamma^{i} \geq 1$ and use $p^{\mathrm{RN}}$ for consensus, and $\exists \hat{\mathcal{I}} \subset \mathcal{I}: P \in \operatorname{Conv}_{i \in \hat{\mathcal{I}}}\left(\pi^{i}\right), \forall i \in \hat{\mathcal{I}}, \alpha^{i} \in(0, \bar{\alpha}]$; then there exists $\gamma \in \Delta^{|\hat{\mathcal{I}}|}$ :

$$
\begin{aligned}
& -\bar{\alpha} \sum_{i \in \hat{\mathcal{I}}} \frac{\gamma^{i}}{\alpha^{i}} \bar{d}\left(P \| p^{i}\right) \\
& \quad=-\bar{\alpha} \sum_{i \in \hat{\mathcal{I}}} \frac{\gamma^{i}}{\alpha^{i}} \bar{d}\left(P \| p^{\mathrm{RN}}\right)+\lim _{t \rightarrow \infty} \frac{\bar{\alpha}}{t} \sum_{\tau=1}^{t} \mathrm{E}_{P}\left[\frac{P}{p_{\tau}^{\mathrm{RN}}}-1\right]-\left|O\left(\bar{\alpha}^{2}\right)\right| .
\end{aligned}
$$

Proof. By assumption, for all $i \in \overline{\mathcal{I}}$ and $(t, \sigma), p^{i}\left(\sigma_{t} \mid\right)=p^{\mathrm{RN}}\left(\sigma_{t} \mid\right)+\alpha^{i}\left(\pi^{i}\left(\sigma_{t}\right)-\right.$ $\left.p^{\mathrm{RN}}\left(\sigma_{t} \mid\right)\right)$.

As we did in Lemma 8, we use Taylor's theorem to exactly approximate

$$
\mathrm{E}_{P}\left[\ln \frac{p_{t}^{i}}{p_{t}^{\mathrm{RN}}}=\mathrm{E}_{P} \ln \left(1+\alpha^{i}\left(\frac{\pi^{i}}{p_{t}^{\mathrm{RN}}}-1\right)\right)\right] \text { around } 1 .
$$

As before, we need to show that the following uniform bounds holds for all $i \in \hat{\mathcal{I}}$ :

(i) $\left(\frac{\pi^{i}\left(\sigma_{t}\right)}{p^{\mathrm{RN}}\left(\sigma_{t} \mid\right)}-1\right)>-1 \quad$ uniformly in $(t, \sigma)$, independent of the $\alpha^{i}$.

(ii) $\left(\frac{\pi^{i}\left(\sigma_{t}\right)}{p^{\mathrm{RN}}\left(\sigma_{t} \mid\right)}-1\right)$ is bounded above uniformly in $(t, \sigma)$ independently of the $\alpha^{i}$ :

$$
\exists K<\infty: \forall(t, \sigma), \forall i \in \hat{\mathcal{I}}, \quad\left(\frac{\pi^{i}\left(\sigma_{t}\right)}{p^{\mathrm{RN}}\left(\sigma_{t} \mid\right)}-1\right)<K .
$$


Proof of (ii). By Proposition 1, all agents in $\mathcal{I} \backslash \overline{\mathcal{I}}$ vanish thus, WLOG, we can consider this economy as being populated only by agents with CRRA utilities with $\gamma^{i} \geq 1$. By Lemma 11, for all $(t, \sigma), p^{\mathrm{RN}}\left(\sigma_{t} \mid\right) \geq \min _{i \in \overline{\mathcal{I}}} \pi^{i}\left(\sigma_{t}\right)$; thus

$$
\forall(t, \sigma), \forall i \in \hat{\mathcal{I}}, \quad \frac{\pi^{i}\left(\sigma_{t}\right)}{p^{\mathrm{RN}}\left(\sigma_{t} \mid\right)}-1<\frac{\max _{i \in \hat{\mathcal{I}}} \pi^{i}\left(\sigma_{t}\right)}{\min _{i \in \overline{\mathcal{I}}} \pi^{i}\left(\sigma_{t}\right)}=K<\text { by Def. } 4 \infty .
$$

Utilising conditions (i) and (ii) as done in Lemma 8, we obtain the exact approximation

$$
\begin{aligned}
& \mathrm{E}_{P}\left[\ln \frac{p_{t}^{i}}{p_{t}^{\mathrm{RN}}}\right]=\mathrm{E}_{P}\left[\ln \left(1+\alpha^{i}\left(\frac{\pi^{i}}{p_{t}^{\mathrm{RN}}}-1\right)\right)\right]=\alpha^{i} \mathrm{E}_{P}\left[\frac{\pi^{i}}{p_{t}^{\mathrm{RN}}}-1\right]-\left|O\left(\left(\alpha^{i}\right)^{2}\right)\right| \\
& \Rightarrow-\bar{d}\left(P \| p^{i}\right)=-\bar{d}\left(P \| p^{\mathrm{RN}}\right)+\lim _{t \rightarrow \infty} \frac{\alpha^{i}}{t} \sum_{\tau=1}^{t} \mathrm{E}_{P}\left[\frac{\pi^{i}}{p_{\tau}^{\mathrm{RN}}}-1\right]-\left|O\left(\left(\alpha^{i}\right)^{2}\right)\right|, \quad P \text {-a.s. }
\end{aligned}
$$

Equation (12) is obtained taking the $\gamma_{\alpha}$ weighted sum of the above expression, as in Lemma 8.

Proof of Theorem 2. In equilibrium, the following inequalities must hold $P$-a.s.:

$$
\begin{aligned}
& \forall i \in \hat{\mathcal{I}}, \quad \bar{d}\left(P \| p^{M}\right)-\bar{d}\left(P \| p^{i}\right) \leq^{\text {By Prop. 2(i) } 0} \\
& \Rightarrow \forall \gamma \in \Delta^{|\hat{\mathcal{I}}|}, \quad \bar{\alpha} \sum_{i \in \hat{\mathcal{I}}} \frac{\gamma^{i}}{\alpha^{i}} \bar{d}\left(P \| p^{M}\right)-\bar{\alpha} \sum_{i \in \hat{\mathcal{I}}} \frac{\gamma^{i}}{\alpha^{i}} \bar{d}\left(P \| p^{i}\right) \leq 0 \\
& \Rightarrow{ }^{\text {By Lem. } 9}\left(\bar{\alpha} \sum_{i \in \hat{\mathcal{I}}} \frac{\gamma^{i}}{\alpha^{i}} \bar{d}\left(P \| p^{M}\right)-\bar{\alpha} \sum_{i \in \hat{\mathcal{I}}} \frac{\gamma^{i}}{\alpha^{i}} \bar{d}\left(P \| p^{\mathrm{RN}}\right)+\lim _{t \rightarrow \infty} \frac{\bar{\alpha}}{t} \sum_{\tau=1}^{t} \mathrm{E}_{P}\left[\frac{P}{p_{\tau}^{\mathrm{RN}}}-1\right]\right) \\
& \quad \leq\left|O\left(\bar{\alpha}^{2}\right)\right| \\
& \Rightarrow{ }^{\text {By Prop. } 6(\mathrm{i}) \bar{d}\left(P \| p^{M}\right)-\bar{d}\left(P \| p^{R N}\right) \geq 0} \lim _{t \rightarrow \infty} \frac{\bar{\alpha}}{t} \sum_{\tau=1}^{t} \mathrm{E}_{P}\left[\frac{P}{p_{\tau}^{\mathrm{RN}}}-1\right] \leq\left|O\left(\bar{\alpha}^{2}\right)\right| \\
& \Rightarrow \lim _{\alpha \rightarrow 0} \bar{d}\left(P \| p_{\bar{\alpha}}^{\mathrm{RN}}\right)=0 .
\end{aligned}
$$

The last implication holds because $p^{\mathrm{RN}}=P$ is the only minimizer of the continuous nonnegative functions $d\left(P \| p_{t}^{\mathrm{RN}}\right)$ and $\mathrm{E}_{P}\left[P / p_{t}^{\mathrm{RN}}-1\right]$.

Lemma 10. Under A1-A5, for all $(t, \sigma)$,

$$
\begin{aligned}
& p^{\mathrm{RN}}\left(\sigma_{t} \mid\right)=\sum_{i \in \mathcal{I}} \rho^{i}\left(\sigma_{t} \mid\right) \psi_{t-1}^{i}, \\
& \text { with } \rho^{i}\left(\sigma_{t} \mid\right):=\frac{p^{i}\left(\sigma_{t} \mid\right)^{\frac{1}{\gamma^{i}}} p^{\mathrm{RN}}\left(\sigma_{t} \mid\right)^{1-\frac{1}{\gamma^{i}}}}{\sum_{\tilde{\sigma}_{t} \in \mathcal{S}} p^{i}\left(\tilde{\sigma}_{t} \mid\right)^{\frac{1}{\gamma^{i}}} p^{\mathrm{RN}}\left(\tilde{\sigma}_{t} \mid\right)^{1-\frac{1}{\gamma^{i}}}} \in \Delta^{S} \text { and } \psi_{t-1}^{i} \in \Delta^{\mathcal{I}} \text {. }
\end{aligned}
$$


Proof. (i) Constant aggregate endowment. Equation (5) in Lemma 6 tells us that, on every equilibrium path,

$$
\forall(t, \sigma), \quad \frac{q\left(\sigma_{t} \mid\right)}{\beta}=\sum_{i \in \mathcal{I}} p^{i}\left(\sigma_{t} \mid\right)^{\frac{1}{\gamma^{i}}}\left(\frac{q\left(\sigma_{t} \mid\right)}{\beta}\right)^{1-\frac{1}{\gamma^{i}}} \phi_{t-1}^{i},
$$

By Definition $8, p^{\mathrm{RN}}\left(\sigma_{t} \mid\right)=q\left(\sigma_{t} \mid\right) / \sum_{\tilde{\sigma}_{t} \in \mathcal{S}} q\left(\tilde{\sigma}_{t} \mid\right)$. Thus, for all $(t, \sigma)$

$$
\begin{aligned}
& \left.p^{\mathrm{RN}}\left(\sigma_{t} \mid\right)=\sum_{i \in \mathcal{I}} p^{i}\left(\sigma_{t} \mid\right)^{\frac{1}{\gamma^{i}}} p^{\mathrm{RN}}\left(\sigma_{t} \mid\right)\right)^{1-\frac{1}{\gamma^{i}}}\left(\frac{\beta}{\sum_{\tilde{\sigma}_{t} \in \mathcal{S}} q\left(\tilde{\sigma}_{t} \mid\right)}\right)^{\frac{1}{\gamma^{i}}} \phi_{t-1}^{i} \\
& =\sum_{i \in \mathcal{I}} \frac{p^{i}\left(\sigma_{t} \mid\right)^{\frac{1}{\gamma^{i}}} p^{\mathrm{RN}}\left(\sigma_{t} \mid\right)^{1-\frac{1}{\gamma^{i}}}}{\sum_{\tilde{\sigma}_{t} \in \mathcal{S}} p^{i}\left(\tilde{\sigma}_{t} \mid\right)^{\frac{1}{\gamma^{i}}} p^{\mathrm{RN}}\left(\tilde{\sigma}_{t} \mid\right)^{1-\frac{1}{\gamma^{i}}}} \sum_{\tilde{\sigma}_{t} \in \mathcal{S}} p^{i}\left(\tilde{\sigma}_{t} \mid\right)^{\frac{1}{\gamma^{i}}} p^{\mathrm{RN}}\left(\tilde{\sigma}_{t} \mid\right)^{1-\frac{1}{\gamma^{i}}}\left(\frac{\beta}{\sum_{\tilde{\sigma}_{t} \in \mathcal{S}} q\left(\tilde{\sigma}_{t} \mid\right)}\right)^{\frac{1}{\gamma^{i}}} \phi_{t-1}^{i} \\
& :=\sum_{i \in \mathcal{I}} \rho^{i}\left(\sigma_{t} \mid\right) \psi_{t-1}^{i},
\end{aligned}
$$

with

$$
\psi_{t-1}:=\sum_{\tilde{\sigma}_{t} \in \mathcal{S}} p^{i}\left(\tilde{\sigma}_{t} \mid\right)^{\frac{1}{\gamma^{i}}} p^{\mathrm{RN}}\left(\tilde{\sigma}_{t} \mid\right)^{1-\frac{1}{\gamma^{i}}}\left(\frac{\beta}{\sum_{\tilde{\sigma}_{t} \in \mathcal{S}} q\left(\tilde{\sigma}_{t} \mid\right)}\right)^{\frac{1}{\gamma^{i}}} \phi_{t-1}^{i} .
$$

Note that $\psi_{t-1}^{i} \in \Delta^{\mathcal{I}}$ because it is independent of $\sigma_{t}$ and

$$
\sum_{\sigma_{t} \in \mathcal{S}} p^{\mathrm{RN}}\left(\sigma_{t} \mid\right)=1 \quad \text { and } \quad \sum_{\sigma_{t} \in \mathcal{S}} \rho^{i}\left(\sigma_{t} \mid\right)=1
$$

(ii) General aggregate endowment and common CRRA parameter $\gamma$

Equation (9) in Lemma 7 tells us that on every equilibrium path,

$$
\begin{aligned}
& \forall(t, \sigma), \\
& \quad \frac{q\left(\sigma_{t} \mid\right)}{\beta}\left(\frac{\mathbf{e}_{t}(\sigma)}{\mathbf{e}_{t-1}(\sigma)}\right)^{\gamma} \\
& \quad=\sum_{i \in \mathcal{I}} p^{i}\left(\sigma_{t} \mid\right)^{\frac{1}{\gamma}}\left(\frac{q\left(\sigma_{t} \mid\right)}{\beta}\left(\frac{\mathbf{e}_{t}(\sigma)}{\mathbf{e}_{t-1}(\sigma)}\right)^{\gamma}\right)^{1-\frac{1}{\gamma}} \phi_{t-1}^{i} p^{i}\left(\sigma_{t} \mid\right)^{\frac{1}{\gamma^{i}}} p^{\mathrm{RN}}\left(\sigma_{t} \mid\right)^{1-\frac{1}{\gamma^{i}}}
\end{aligned}
$$

By Definition 8, $p_{\gamma}^{\mathrm{RN}}\left(\sigma_{t} \mid \sigma^{t-1}\right)=q\left(\sigma_{t} \mid \sigma^{t-1}\right) \mathbf{e}_{t}(\sigma)^{\gamma} / \sum_{\tilde{\sigma}_{t}} q\left(\tilde{\sigma}_{t} \mid \sigma^{t-1}\right) \mathbf{e}_{t}(\tilde{\sigma})^{\gamma}$.

Thus, for all $(t, \sigma)$,

$$
p_{\gamma}^{\mathrm{RN}}\left(\sigma_{t} \mid\right)=\sum_{i \in \mathcal{I}} p^{i}\left(\sigma_{t} \mid\right)^{\frac{1}{\gamma}} p_{\gamma}^{\mathrm{RN}}\left(\sigma_{t} \mid\right)^{1-\frac{1}{\gamma}}\left(\frac{\beta\left(\mathbf{e}_{t-1}(\sigma)\right)^{\gamma}}{\sum_{\tilde{\sigma} \in \mathcal{S}} q(\tilde{\boldsymbol{\sigma}} \mid)\left(\mathbf{e}_{t}(\tilde{\sigma})\right)^{\gamma}}\right)^{\frac{1}{\gamma}} \phi_{t-1}^{i}
$$




$$
\begin{aligned}
= & \sum_{i \in \mathcal{I}} p^{i}\left(\sigma_{t}\right)^{\frac{1}{\gamma}} \frac{p_{\gamma}^{\mathrm{RN}}\left(\sigma_{t} \mid\right)^{1-\frac{1}{\gamma}}}{\sum_{\tilde{\sigma}_{t} \in \mathcal{S}} p^{i}\left(\tilde{\sigma}_{t} \mid\right)^{\frac{1}{\gamma}} p_{\gamma}^{\mathrm{RN}}\left(\tilde{\sigma}_{t} \mid\right)^{1-\frac{1}{\gamma}}} \\
& \times \sum_{\tilde{\sigma}_{t} \in \mathcal{S}} p^{i}\left(\tilde{\sigma}_{t} \mid\right)^{\frac{1}{\gamma}} p_{\gamma}^{\mathrm{RN}}\left(\tilde{\sigma}_{t} \mid\right)^{1-\frac{1}{\gamma}}\left(\frac{\beta\left(\mathbf{e}_{t-1}(\sigma)\right)^{\gamma}}{\sum_{\tilde{\sigma}_{t} \in \mathcal{S}} q(\tilde{\sigma} \mid)\left(\mathbf{e}_{t}\left(\tilde{\sigma}_{t}\right)\right)^{\gamma}}\right)^{\frac{1}{\gamma}} \phi_{t-1}^{i} \\
= & \sum_{i \in \mathcal{I}} \rho^{i}\left(\sigma_{t} \mid\right) \psi_{t}^{i}
\end{aligned}
$$

with $\psi_{t}:=\sum_{\tilde{\sigma}_{t} \in \mathcal{S}} p^{i}\left(\tilde{\sigma}_{t} \mid\right)^{\frac{1}{\gamma}} p_{\gamma}^{\mathrm{RN}}\left(\tilde{\sigma}_{t} \mid\right)^{1-\frac{1}{\gamma}}\left(\frac{\beta\left(\mathbf{e}_{t-1}(\sigma)\right)^{\gamma}}{\sum_{\tilde{\sigma}_{t} \in \mathcal{S}} q\left(\tilde{\sigma}_{t} \mid\right)\left(\mathbf{e}_{t}(\tilde{\sigma})\right)^{\gamma}}\right)^{\frac{1}{\gamma}} \phi_{t-1}^{i} \in \Delta^{\mathcal{I}}$, as shown above for (i).

LEмма 11. Under A1-A5, if agents' utilities are CRRA with $\gamma^{i} \geq 1$ for all $i \in \mathcal{I}$, then

$$
\forall(t, \sigma), \quad p^{\mathrm{RN}}\left(\sigma_{t} \mid\right) \geq \min _{i \in \mathcal{I}}\left\{\pi^{i}\left(\sigma_{t}\right)\right\} .
$$

Proof. By Lemma 10, for all $(t, \sigma), \exists \psi_{t} \in \Delta^{I}$ such that

$$
p^{\mathrm{RN}}\left(\sigma_{t} \mid\right)=\sum_{i \in \mathcal{I}} \rho^{i}\left(\sigma_{t} \mid\right) \psi_{t-1}^{i}, \quad \text { with } \rho^{i}\left(\sigma_{t} \mid\right)=\frac{p^{i}\left(\sigma_{t} \mid\right)^{\frac{1}{\gamma^{i}}} p^{\mathrm{RN}}\left(\sigma_{t} \mid\right)^{1-\frac{1}{\gamma^{i}}}}{\sum_{\tilde{\sigma}_{t} \in \mathcal{S}} p^{i}\left(\tilde{\sigma}_{t} \mid\right)^{\frac{1}{\gamma^{i}}} p^{\mathrm{RN}}\left(\tilde{\sigma}_{t} \mid\right)^{1-\frac{1}{\gamma^{i}}}} .
$$

Notice that for all $i \in \mathcal{I}, \gamma^{i} \geq 1$ implies that

$$
\begin{aligned}
\forall i \in \mathcal{I}, \forall(t, \sigma), \quad \rho^{i}\left(\sigma_{t} \mid\right) & =\frac{p^{i}\left(\sigma_{t} \mid\right)^{\frac{1}{\gamma^{i}}} p^{\mathrm{RN}}\left(\sigma_{t} \mid\right)^{1-\frac{1}{\gamma^{i}}}}{\sum_{\tilde{\sigma}_{t} \in \mathcal{S}} p^{i}\left(\tilde{\sigma}_{t} \mid\right)^{\frac{1}{\gamma^{i}}} p^{\mathrm{RN}}\left(\tilde{\sigma}_{t} \mid\right)^{1-\frac{1}{\gamma^{i}}}} \\
& \geq{ }^{(i)} p^{i}\left(\sigma_{t} \mid\right)^{\frac{1}{\gamma^{i}}} p^{\mathrm{RN}}\left(\sigma_{t} \mid\right)^{1-\frac{1}{\gamma^{i}}} \\
& \geq{ }^{(i i)} \min \left\{p^{\mathrm{RN}}\left(\sigma_{t} \mid\right), p^{i}\left(\sigma_{t} \mid\right)\right\} \\
& =\min \left\{p^{\mathrm{RN}}\left(\sigma_{t} \mid\right),\left(1-\alpha^{i}\right) p^{\mathrm{RN}}\left(\sigma_{t} \mid\right)+\alpha^{i} \pi^{i}\left(\sigma_{t} \mid\right)\right\} \\
& \geq \min \left\{p^{\mathrm{RN}}\left(\sigma_{t} \mid\right), \pi^{i}\left(\sigma_{t} \mid\right)\right\},
\end{aligned}
$$

with equality if and only if $p^{\mathrm{RN}}\left(\sigma_{t} \mid\right)=\pi^{i}\left(\sigma_{t}\right)$.

(i) The ordering of geometric and arithmetic means implies

$$
\begin{aligned}
\forall i & \in \mathcal{I}, \forall(t, \sigma), \quad p^{i}\left(\sigma_{t} \mid\right)^{\frac{1}{\gamma^{i}}} p^{\mathrm{RN}}\left(\sigma_{t} \mid\right)^{1-\frac{1}{\gamma^{i}}} \leq\left(\frac{1}{\gamma^{i}}\right) p^{i}\left(\sigma_{t} \mid\right)+\left(1-\frac{1}{\gamma^{i}}\right) p^{\mathrm{RN}}\left(\sigma_{t} \mid\right) \\
& \Rightarrow \sum_{\tilde{\sigma}_{t} \in \mathcal{S}} p^{i}\left(\tilde{\sigma}_{t} \mid\right)^{\frac{1}{\gamma^{i}}} p^{\mathrm{RN}}\left(\tilde{\sigma}_{t} \mid\right)^{1-\frac{1}{\gamma^{i}}} \leq 1 ;
\end{aligned}
$$


(ii) A property of the geometric mean.

We continue by contradiction assuming that

$$
H 1: \quad \exists \sigma_{t}^{\prime}: p^{\mathrm{RN}}\left(\sigma_{t}^{\prime} \mid\right)<\min _{i \in \mathcal{I}}\left\{\pi^{i}\left(\sigma_{t}^{\prime} \mid\right)\right\} .
$$

This is absurd because

$$
p^{\mathrm{RN}}\left(\sigma_{t}^{\prime} \mid\right)={ }^{\text {By Lem. }} 10 \sum_{i \in \mathcal{I}} \rho^{i}\left(\sigma_{t}^{\prime} \mid\right) \psi_{t-1}^{i} \geq^{\text {By } H 1: \text { and Eq. (13) }} p^{\mathrm{RN}}\left(\sigma_{t}^{\prime} \mid\right),
$$

with equality if only if $p^{\mathrm{RN}}\left(\sigma_{t} \mid\right)=p^{i}\left(\sigma_{t} \mid\right)=\pi^{i}\left(\sigma_{t}\right)$ for all $i$ with positive weight $\psi_{t}^{i}$, a contradiction to $H 1$.

\section{ApPendix C: Proof of Competitive equilibrium eXISTence}

We define a competitive equilibrium with consensus as a $2 I+2$-tuple of sequences of consumption allocations $\left(c_{t}^{i}(\sigma)\right)_{t=0}^{\infty}$, beliefs $\left(p^{i}\left(\sigma_{t} \mid\right)\right)_{t=0}^{\infty}$, consensus beliefs $\left(p^{C}\left(\sigma_{t} \mid\right)\right)_{t=0}^{\infty}$ and prices $\left(q\left(\sigma^{t}\right)\right)_{t=0}^{\infty}$, one for each $\sigma \in \Sigma$, such that:

1. Each agent $i \in \mathcal{I}$ consumption solves the utility maximization given endogenous beliefs $p^{i}$ and prices $\left(q\left(\sigma^{t}\right)\right)_{\left(t, \sigma^{t}\right)}$

$$
\max _{\left(c_{t}^{i}(\sigma)\right)_{t=0}^{\infty}} \mathrm{E}_{p^{i}}\left[\sum_{t=0}^{\infty} \beta^{t} u^{i}\left(c_{t}^{i}(\sigma)\right)\right] \text { s.t. } \sum_{t \geq 0} \sum_{\sigma^{t} \in \Sigma^{t}} q\left(\sigma^{t}\right)\left(c_{t}^{i}(\sigma)-e_{t}^{i}(\sigma)\right) \leq 0 .
$$

2. All good markets clear:

$$
\sum_{i \in \mathcal{I}} c_{t}^{i}(\sigma)=\sum_{i \in \mathcal{I}} e_{t}^{i}(\sigma) \text { for all }(t, \sigma) .
$$

3. Each agent $i \in \mathcal{I}$ beliefs $p^{i}$ are as in Definition 4 for a given choice of consensus belief $p^{C}$ and idiosyncratic belief $\pi^{i}$.

4. The consensus belief $p^{C}$ is $p^{M}$ as in Definition 7 or $p^{\mathrm{RN}}$ as in Definition 8 or $p_{\gamma}^{\mathrm{RN}}$ as in Definition 9.

The competitive equilibrium with consensus differs from the standard one in that agents' beliefs are endogenously determined.

In what follows, we prove that under A1-A4 (A5) there exists a competitive equilibrium with consensus. In the first step, we assign an initial consumption share distribution $\phi_{0}$ and derive sequences of consumption, prices, individual beliefs, and consensus beliefs consistent with the First-Order Conditions (FOC) of agents' utility maximization problem, with market clearing, and with the definition of individual and consensus beliefs. This step is similar to the computation of a Pareto optimal allocation given a set of Pareto weights but, due to the endogeneity of beliefs, involves an additional fixed point argument for each iteration. The Brouwer fixed-point theorem, together with the 
smoothness of our maps, guarantees the existence of such a fixed point for each iteration. The details of this step are different for $p^{M}, p^{\mathrm{RN}}$, and $p_{\gamma}^{\mathrm{RN}}$ because of their different analytic form.

In the second step, we show that there exists an initial distribution of consumption shares such that each agent's budget constraint is satisfied. The main difference between this step and the standard proof of the existence of the competitive equilibrium with exogenous beliefs is that in our case the initial consumption shares distribution affects prices also via its effect on beliefs. This further complication does not change the typical argument. Even in this case, Brouwer's fixed-point theorem guarantees the existence of a fixed point.

Remark. Our proof ensures existence, not uniqueness. Multiplicity of equilibria is not problematic because our results hold in all the equilibria that exist.

Let us start from the system of FOCs. Having defined $\bar{c}_{t}^{i}(\sigma)=1 / u^{i \prime}\left(c_{t}^{i}(\sigma)\right)$, the system of agent $i \mathrm{FOC}$ and his budget constraint is

$$
\left\{\begin{array}{l}
\bar{c}_{0}^{i}=\frac{1}{\lambda^{i}}, \\
\bar{c}_{t}^{i}(\sigma)=\frac{\beta p^{i}\left(\sigma_{t} \mid\right)}{q\left(\sigma_{t} \mid\right)} \bar{c}_{t-1}^{i}(\sigma) \quad \text { for all }(t, \sigma), \\
\sum_{t \geq 0} \sum_{\sigma^{t} \in \Sigma^{t}} q\left(\sigma^{t}\right)\left(c_{t}^{i}(\sigma)-e_{t}^{i}(\sigma)\right)=0,
\end{array}\right.
$$

where $\lambda^{i}$ is the multiplier associated with agent $i$ 's budget constraint.

First step $-p^{M}$ is the consensus used by all $i \in \mathcal{I} \quad$ By Lemma 5 for all $(t, \sigma)$,

$$
p^{M}\left(\sigma_{t} \mid\right)=\sum_{i \in \mathcal{I}} \pi^{i} \frac{\alpha^{i} \bar{c}_{t-1}^{i}(\sigma)}{\sum_{j \in \mathcal{I}} \alpha^{j} \bar{c}_{t-1}^{j}(\sigma)}
$$

so that, using Definition 4,

$$
p^{i}\left(\sigma_{t} \mid\right)=\left(1-\alpha^{i}\right) \sum_{j \in \mathcal{I}} \pi^{j} \frac{\alpha^{j} \bar{c}_{t-1}^{j}(\sigma)}{\sum_{k \in \mathcal{I}} \alpha^{k} \bar{c}_{t-1}^{k}(\sigma)}+\alpha^{i} \pi^{i} .
$$

Thus, for each given initial consumption distribution $\left\{\phi_{0}^{i}\right\}_{i=1}^{I}$ we can compute initial marginal utilities $\left\{\bar{c}_{0}^{i}\right\}_{i=1}^{I}$, consensus beliefs $p_{1}^{M}$, and individual beliefs $\left\{p_{1}^{i}\right\}_{i=1}^{I}$.

Having determined beliefs, we can proceed to compute equilibrium consumption in date $t=1$ as follows. From the second equation of (14), the ratio of agent $i=1$ to agent $j$ FOC between $t=0$ and $\left(t=1, \sigma_{1}\right)$ gives

$$
\left(u^{j^{\prime}}\right)^{-1}\left(\frac{\bar{c}_{0}^{1}}{\bar{c}_{0}^{j}} \frac{p^{1}\left(\sigma_{1} \mid\right)}{p^{j}\left(\sigma_{1} \mid\right)} u^{1 \prime}\left(\phi_{1}^{1}\left(\sigma_{1}\right) e_{1}\left(\sigma_{1}\right)\right)\right)=\phi_{1}^{j}\left(\sigma_{1}\right) e_{1}\left(\sigma_{1}\right) .
$$


Aggregating over agents, we find

$$
\sum_{j \in \mathcal{I}}\left(u^{j^{\prime}}\right)^{-1}\left(\frac{\bar{c}_{0}^{1}}{\bar{c}_{0}^{j}} \frac{p^{1}\left(\sigma_{1} \mid\right)}{p^{j}\left(\sigma_{1} \mid\right)} u^{1 \prime}\left(\phi_{1}^{1}\left(\sigma_{1}\right) e_{1}\left(\sigma_{1}\right)\right)\right)=e_{1}\left(\sigma_{1}\right) .
$$

Agent $i=1$ consumption share $\phi_{1}^{1}\left(\sigma_{1}\right)$ can be derived from the above. A solution $\phi_{1}^{1}\left(\sigma_{1}\right)$ of (16) always exists in $(0,1)$ because, by A1, A3, the l.h.s. is continuous in $\phi_{1}^{1}\left(\sigma_{1}\right)=x$, goes to $0<e_{1}\left(\sigma_{1}\right)$ for $x \rightarrow 0$, and is larger than $e_{1}\left(\sigma_{1}\right)$ in $x=1 .^{11}$ Repeating the same argument for all the agents, we find $\left\{\phi_{1}^{i}\left(\sigma_{1}\right)\right\}_{i=1}^{I}$ and, repeating for all $\sigma_{1} \in \mathcal{S}$, we find $\left\{\phi_{1}^{i}\right\}_{i=1}^{I}$. Iterating these steps for all $t$ and for all $\sigma^{t}$ gives the stream of individual consumptions, individual beliefs, and consensus beliefs for each choice of path $\sigma \in \Sigma$ and for each choice of $\left\{\phi_{0}^{i}\right\}_{i=1}^{I}$.

First step $-p^{R N}$ is the consensus used by all $i \in \mathcal{I} \quad$ By Lemma 2, in $t=0$ the consensus $p^{\mathrm{RN}}$ in $\left(t=1, \sigma_{1}\right)$ defined in (8) can be written as

$$
p^{\mathrm{RN}}\left(\sigma_{1} \mid\right)=\frac{\sum_{i \in \mathcal{I}} p^{i}\left(\sigma_{1} \mid\right) \frac{\bar{c}_{0}^{i}}{\sum_{j \in \mathcal{I}} \bar{c}_{1}^{j}\left(\sigma_{1}\right)}}{\sum_{\tilde{\sigma}_{1} \in \mathcal{S}} \sum_{i \in \mathcal{I}} p^{i}\left(\tilde{\sigma}_{1} \mid\right) \frac{\bar{c}_{0}^{i}}{\sum_{j \in \mathcal{I}} \bar{c}_{1}^{j}\left(\tilde{\sigma}_{1}\right)}} \quad \forall \sigma_{1} \in \mathcal{S},
$$

where $p^{\mathrm{RN}}\left(\sigma_{1} \mid\right)$ is also on the r.h.s. in each individual belief $p^{i}\left(\sigma_{1} \mid\right)$, for all $i \in \mathcal{I}$. The above and (16) for all $\sigma_{1}$ define a map from $\Delta^{S}$ to $\Delta^{S}$ as follows. For each given $\rho \in \Delta^{S}$, (16) for all $\sigma_{1}$ and all $i$ allows to compute $\left\{c_{1}^{i}(\rho)\right\}_{i=1}^{I}$ when individual beliefs $\left\{p_{1}^{i}\right\}_{i=1}^{I}$ are built using $\rho$ as the consensus, $\left\{p_{1}^{i}(\rho)\right\}_{i=1}^{I}$. Then, having consumption $\left\{c_{1}^{i}(\rho)\right\}_{i=1}^{I=1}$ and beliefs $\left\{p_{1}^{i}(\rho)\right\}_{i=1}^{I}$, (17) gives the consensus beliefs $p_{1}^{\mathrm{RN}}(\rho)$.

The equilibrium consensus $\rho$ solves $p_{1}^{\mathrm{RN}}(\rho)=\rho$. Its existence follows from Brouwer's fixed-point theorem: the map built composing (16) and (17) goes from $\Delta^{S}$ to $\Delta^{S}$ and is continuous. To prove continuity note that, given $\rho$, for each $i$ and $\sigma_{1}$ (16) defines a function $F^{i}\left(\rho, \phi_{1}^{i}\left(\sigma_{1}\right)\right)$ such that the solution of

$$
F_{i}\left(\rho, \phi_{1}^{i}\left(\sigma_{1}\right)\right)=0 \quad \text { determines } c_{1}^{i}\left(\sigma_{1}\right)(\rho)=e_{1}\left(\sigma_{1}\right) \phi_{1}^{i}\left(\sigma_{1}\right) .
$$

Continuity of $c_{1}^{i}\left(\sigma_{1}\right)(\rho)$ in $\rho$ follows from the implicit function theorem because, by $\mathrm{A} 1, \mathrm{~A} 3, F_{i}(\rho, x)$ is the sum of compositions of monotone functions, and thus monotone, implying that the derivative $\partial F_{i} / \partial x$ is different from zero in the solution $\phi_{1}^{i}\left(\sigma_{1}\right)$ of $F_{i}(\rho, x)=0$. Continuity of the composed map follows from continuity of $c_{1}^{i}\left(\sigma_{1}\right)(\rho)$ for all $i$ and $\sigma_{1}$ and from continuity of (17).

${ }^{11}$ For the latter, note that

$$
\left.\sum_{j \in \mathcal{I}}\left(u^{j^{\prime}}\right)^{-1}\left(\frac{\bar{c}_{0}^{1}}{\bar{c}_{0}^{j}} \frac{p^{1}\left(\sigma_{1} \mid\right)}{p^{j}\left(\sigma_{1} \mid\right)} u^{1 \prime}\left(\phi_{1}^{1}\left(\sigma_{1}\right) e_{1}\left(\sigma_{1}\right)\right)\right)\right|_{\phi_{1}^{1}\left(\sigma_{1}\right)=1}=\sum_{j \neq 1}\left(u^{j^{\prime}}\right)^{-1}\left(\frac{\bar{c}_{0}^{1}}{\bar{c}_{0}^{j}} \frac{p^{1}\left(\sigma_{1} \mid\right)}{p^{j}\left(\sigma_{1} \mid\right)} u^{1 \prime}\left(e_{1}\left(\sigma_{1}\right)\right)\right)+e_{1}\left(\sigma_{1}\right) .
$$


Having found the date $t=0$ consensus beliefs $p_{1}^{\mathrm{RN}}$, the corresponding date $t=1$ consumption distribution and individual beliefs are $\left\{c_{1}^{i}\left(p_{1}^{\mathrm{RN}}\right)\right\}_{i=1}^{I}$ and $\left\{p_{1}^{i}\left(p_{1}^{\mathrm{RN}}\right)\right\}_{i=1}^{I}$, respectively. Iterating these steps for all $t$ and for all $\sigma^{t}$ gives a sequence of consumptions, individual and consensus beliefs as a function of the initial consumption distribution $\phi_{0}$.

First step $-p_{\gamma}^{\mathrm{RN}}$ is the consensus used by all $i \in \mathcal{I} \quad$ When the consensus beliefs is $p_{\gamma}^{\mathrm{RN}}$, as in (9), this first step of the proof is the same provided that map (17) is replaced by the corresponding expression of $p_{\gamma}^{\mathrm{RN}}$ as a function of equilibrium consumption derived in Lemma 3.

First step-different agents use different consensuses The computation of streams of consumption, individual beliefs, and consensus beliefs given an initial consumption distribution $\phi_{0}$ can be performed also when different agents use different consensuses. We consider two cases: (i) agents use either $p^{M}$ or $p^{\mathrm{RN}}$; (ii) agents use either $p^{M}$ or $p_{\gamma}^{\mathrm{RN}} \cdot 12$

When agents use either $p^{M}$ or $p^{\mathrm{RN}}$, the proof proceeds similar to when all agents use only $p^{\mathrm{RN}}$. In $t=0$, given a candidate consensus beliefs $\rho \in \Delta^{S}$, initial individual beliefs of those mixing with $p^{\mathrm{RN}}$ are computed directly from $\rho$ while individual beliefs of those mixing with $p^{M}$ are computed using (15) with $\pi^{j}=p^{j}$ if $j$ chooses $p^{\mathrm{RN}}$ as consensus. Having all agents' individual beliefs for a given $\rho$, the combination of (16) and (17) for all $s \in \mathcal{S}$ determines the fixed point $\rho$ such that $p_{1}^{\mathrm{RN}}(\rho)=\rho$. From here, we proceed as above.

The case when agents use either $p^{M}$ or $p_{\gamma}^{R M}$ proceeds along the same way provided that the map (17) is replaced by the corresponding expression of $p_{\gamma}^{\mathrm{RN}}$ as a function of equilibrium consumption as in Lemma 3.

Second step As shown in the first step, we can derive a stream of individual consumption and beliefs for each given initial consumption distribution $\phi_{0}$. Using the FOC, to such consumption streams there corresponds a sequence of state contingent prices $\left(q\left(\sigma^{t}\right)\right)_{\left(t, \sigma^{t}\right)}$. We have an equilibrium when $\phi_{0}$ is chosen such that all agents' budget constraints, third equation in (14), are satisfied.

Equivalently, define

$$
\begin{aligned}
f_{i}\left(\phi_{0}\right) & =\sum_{t \geq 0} \sum_{\sigma^{t} \in \Sigma^{t}} q\left(\sigma^{t}\right) e_{t}^{i}\left(\sigma^{t}\right)-\sum_{t \geq 0} \sum_{\sigma^{t} \in \Sigma^{t}} q\left(\sigma^{t}\right) e_{t}\left(\sigma^{t}\right) \phi_{t}^{i}\left(\sigma^{t}\right), \\
& \vdots=\vdots=\vdots \\
f_{I}\left(\phi_{0}\right) & =\sum_{t \geq 0} \sum_{\sigma^{t} \in \Sigma^{t}} q\left(\sigma^{t}\right) e_{t}^{I}\left(\sigma^{t}\right)-\sum_{t \geq 0} \sum_{\sigma^{t} \in \Sigma^{t}} q\left(\sigma^{t}\right) e_{t}\left(\sigma^{t}\right) \phi_{t}^{I}\left(\sigma^{t}\right) ;
\end{aligned}
$$

${ }^{12}$ In each case, the definition of competitive equilibrium with consensus should be changed accordingly. In 3 , each agent should be allowed to use the consensus he chooses. In 4 , both consensuses, $p^{M}$ or $p^{\mathrm{RN}}$ in (i) and $p^{M}$ or $p_{\gamma}^{\mathrm{RN}}$ in (ii), should be determined in equilibrium. 
we have a competitive equilibrium with consensus if we can find $\phi \in \Delta^{I}$ such that $f(\phi)=$ 0 . The existence of (at least) one of these points follows from Brouwer's fixed-point theorem, as follows.

First, note that each function is well-defined and continuous in $\phi_{0}$. Well-defined because the aggregate endowment is bounded (A2) and state prices go to zero as fast as $\beta^{t}$ (FOC and A1-A4). Continuous because, as shown in the proof of the first step for $p^{\mathrm{RN}}$, the equilibrium consumption that solves (16) for all $i, t$, and $\sigma_{t}$ is continuous in its parameters (we have proved continuity with respect to $\rho$ but the argument is the same for continuity in $\phi_{0}$, monotonicity in the unknown consumption allows to use the implicit function theorem).

Define the function $f^{+}: \Delta^{I} \rightarrow[0, \infty)^{I}$ as

$$
f_{i}^{+}(\phi)=\max \left\{f_{i}(\phi), 0\right\} \quad \text { for all } i \in \mathcal{I} .
$$

Denote

$$
\nu(\phi)=1+\sum_{i \in \mathcal{I}} f_{i}^{+}(\phi) .
$$

By construction, $\nu(\phi) \geq 1$ for all $\phi \in \Delta^{I}$. Define the function $F: \Delta^{I} \rightarrow \Delta^{I}$ as

$$
F(\phi)=\frac{\phi+f^{+}(\phi)}{\nu(\phi)} .
$$

Continuity of $f_{i}$ for all $i \in \mathcal{I}$ imply that the function $F$ is continuous on the compact convex set $\Delta^{I}$, and thus has a fixed point $\bar{\phi}$ by Brouwer's fixed-point theorem. Showing that $f(\bar{\phi})=0$ ends the proof.

$F(\bar{\phi})=\bar{\phi}$ implies

$$
\frac{\bar{\phi}+f^{+}(\bar{\phi})}{1+\sum_{i \in \mathcal{I}} f_{i}^{+}(\bar{\phi})}=\bar{\phi} \Rightarrow f^{+}(\bar{\phi})=\bar{\phi}\left(\sum_{i \in \mathcal{I}} f_{i}^{+}(\bar{\phi})\right) .
$$

Assume first that $\sum_{i \in \mathcal{I}} f_{i}^{+}(\bar{\phi})>0$. If $\bar{\phi}_{i}=0$, then, by construction, the budget constraint does not hold for $i$ and $f_{i}(\bar{\phi})>0$, so that $f_{i}^{+}(\bar{\phi})>0$, too, leading to a contradiction with (18). Then it must be $\bar{\phi}_{i}>0$ for all $i$, implying $f_{i}^{+}(\bar{\phi})>0$ for all $i$, by (18), and leading to a contradiction with $\sum_{i \in \mathcal{I}} f_{i}(\phi)=0$ for all $\phi$ (Walras' law). It follows that $\sum_{i \in \mathcal{I}} f_{i}^{+}(\bar{\phi})=0$, and thus, being the sum of nonnegative functions, $f_{i}^{+}(\bar{\phi})=0$ for all $i$, implying $f_{i}(\bar{\phi}) \leq 0$ for all $i$. The latter together with $\sum_{i \in \mathcal{I}} f_{i}(\bar{\phi})=0$ (Walras' law) implies $f_{i}(\bar{\phi})=0$ for all $i \in \mathcal{I}$.

\section{REFERENCES}

Alchian, Armen A. (1950), “Uncertainty, evolution, and economic theory.” Journal of Political Economy, 58, 211-221. [1630]

Ali, Mukhtar M. (1977), "Probability and utility estimates for racetrack bettors." Journal of Political Economy, 85, 803-815. [1630] 
Arrow, Kenneth J., Robert Forsythe, Micheal Gorham, Robert Hahn, Robin Hanson, John O. Ledyard, Saul Levmore, Robert Litan, Paul Milgrom, Forrest D. Nelson, George R. Neumann, Marco Ottaviani, Thomas C. Schelling, Robert J. Shiller, Veron L. Smith, Erik Snowberg, Cass R. Sunstein, Paul C. Tetlock, Philip E. Tetlock, Hal R. Varian, Justin Wolfers, and Eric Zitzewitz (2008), “The promise of prediction markets.” Science, 320, 877-878. [1630]

Aumann, Robert J. (1976), “Agreeing to disagree." Annals of Statistics, 4, 1236-1239. [1629]

Beker, Pablo F. and Subir Chattopadhyay (2010), "Consumption dynamics in general equilibrium: A characterisation when markets are incomplete." Journal of Economic Theory, 145, 2133-2185. [1630]

Berk, Robert (1966), "Limiting behavior of posterior distributions when the model is incorrect.” Annals of Mathematical Statistics, 37, 51-58. [1633]

Blume, Lawrence and David Easley (1992), "Evolution and market behavior." Journal of Economic Theory, 58, 9-40. [1632]

Blume, Lawrence E. and David Easley (2006), "If you are so smart why aren't you rich? Belief selection in complete and incomplete markets.” Econometrica, 74, 929-966. [1628]

Blume, Lawrence E. and David Easley (2009), "The market organism: Long-run survival in markets with heterogenous traders." Journal of Economic Dynamics and Control, 33, 1023-1035. [1630, 1638]

Borovička, Jaroslav (2020), "Survival and long-run dynamics with heterogeneous beliefs under recursive preferences." Journal of Political Economy, 128, 206-251. [1630, 1643]

Bottazzi, Giulio and Pietro Dindo (2014), "Evolution and market behavior with endogenous investment rules." Journal of Economic Dynamics and Control, 48, 121-146. [1630]

Bottazzi, Giulio, Pietro Dindo, and Daniele Giachini (2018), "Long-run heterogeneity in an exchange economy with fixed-mix traders." Economic Theory, 66, 407-447. [1630]

Bottazzi, Giulio and Daniele Giachini (2019), "Far from the madding crowd: Collective wisdom in prediction markets." Quantitative Finance, 19, 1461-1471. [1630]

Breitmayer, Bastian, Filippo Massari, and Matthias Pelster (2019), "Swarm intelligence? Stock opinions of the crowd and stock returns." International Review of Economic and Finance, 64, 443-464. [1630]

Cao, D. (2017), "Speculation and financial wealth distribution under belief heterogeneity.” The Economic Journal, 128, 2258-2281. [1630]

Chen, Hailiang, Prabuddha De, Yu Jeffrey Hu, and Byoung-Hyoun Hwang (2014), "Wisdom of crowds: The value of stock opinions transmitted through social media." Review of Financial Studies, 27, 1367-1403. [1630]

Cogley, Timothy, Thomas J. Sargent, and Viktor Tsyrennikov (2013), "Wealth dynamics in a bond economy with heterogeneous beliefs.” Economic Journal, 124, 1-30. [1630] 
Dindo, Pietro (2019), “Survival in speculative markets." Journal of Economic Thoery, 181, $1-43 .[1630,1643]$

Friedman, Milton (1953), Essays in Positive Economics, volume 231. University of Chicago Press. [1630]

Galton, Francis (1907), "Vox populi (the wisdom of crowds).” Nature, 75, 450-451. [1630]

Geanakoplos, John D. and Heraklis Polemarchakis (1982), "We can't disagree forever." Journal of Economic Theory, 28, 192-200. [1629]

Golub, Benjamin and Matthew O. Jackson (2010), "Naive learning in social networks and the wisdom of crowds." American Economic Journal: Microeconomics, 2, 112-149. [1630, 1633]

Grossman, Sanford J. (1976), "On the efficiency of competitive stock markets where trades have diverse information.” Journal of Finance, 31, 573-585. [1629]

Grossman, Sanford J. (1978), "Further results on the informational efficiency of competitive stock markets.” Journal of Economic Theory, 18, 81-101. [1629]

Grossman, Sanford J. and Joseph E. Stiglitz (1980), “On the impossibility of informationally efficient markets.” American Economic Review, 70, 393-408. [1629]

Guerdjikova, Ani and Emanuela Sciubba (2015), "Survival with ambiguity." Journal of Economic Theory, 155, 50-94. [1630]

Hong, Lu and Scott E. Page (2004), "Groups of diverse problem solvers can outperform groups of high-ability problem solvers.” Proceedings of the National Academy of Sciences of the United States of America, 101, 16385-16389. [1630]

Jadbabaie, Ali, Pooya Molavi, Alvaro Sandroni, and Alireza Tahbaz-Salehi (2012), "NonBayesian social learning." Games and Economic Behavior, 76, 210-225. [1630, 1633]

Jouini, Elyès and Clotilde Napp (2011), "Unbiased disagreement in financial markets, waves of pessimism and the risk-return trade-off." Review of Finance, 15, 575-601. [1630]

Kets, Willemien, David M. Pennock, Rajiv Sethi, and Nisarg Shah (2014), "Betting strategies, market selection, and the wisdom of crowds." In: Twenty-Eighth AAAI Conference on Artificial Intelligence, 735-741. [1630]

Lakonishok, Josef, Andrei Shleifer, and Robert W. Vishny (1992), "The impact of institutional trading on stock prices.” Journal of Financial Economics, 32, 23-43. [1633]

MacLean, Leonard C., Edward O. Thorp, and William T. Ziemba (2011), The Kelly Capital Growth Investment Criterion: Theory and Practice, volume 3. World Scientific. [1633]

Mailath, George J. and Alvaro Sandroni (2003), "Market selection and asymmetric information." Review of Economic Studies, 70, 343-368. [1630]

Manski, Charles F. (2006), "Interpreting the predictions of prediction markets." Economic Letters, 91, 425-429. [1630, 1633] 
Massari, Filippo (2013), “Comment on if you're so smart, why aren't you rich? Belief selection in complete and incomplete markets." Econometrica, 81, 849-851. [1630]

Massari, Filippo (2017), "Markets with heterogeneous beliefs: A necessary and sufficient condition for a trader to vanish." Journal of Economic Dynamics and Control, 78, 190205. [1643, 1648, 1653]

Massari, Filippo (2018), "Price probabilities: A class of Bayesian and non-Bayesian prediction rules.” Economic Theory, 1-34. [1643]

Ottaviani, Marco and Peter Normam Sørensen (2015), "Price reaction to information with heterogeneous beliefs and wealth effects: Underreaction, momentum, and reversal." American Economic Review, 105, 1-34. [1630]

Page, Scott E. (2007), The Difference: How the Power of Diversity Creates Better Groups, Firms, Schools, and Societies. Princeton University Press. [1630]

Radner, Roy (1979), "Rational expectations equilibrium: Generic existence and the information revealed by prices." Econometrica, 47, 655-678. [1629]

Rényi, Alfréd (1961), "On measures of entropy and information.” Fourth Berkeley Symposium on Mathematical Statistics and Probability, 1, 547-561. [1652]

Rubinstein, Mark (1974), "An aggregation theorem for securities markets.” Journal of Financial Economics, 1, 225-244. [1635]

Sandroni, Alvaro (2000), "Do markets favor agents able to make accurate predictions." Econometrica, 68, 1303-1341. [1628, 1630, 1632, 1648]

Shiller, Robert J. (1999), "Human behavior and the efficiency of the financial system.” In Handbook of Macroeconomics, volume 1 (John B. Taylor and Michael Woodford, eds.), 1305-1340, Elsevier. [1633]

Surowiecki, James (2005), The Wisdom of Crowds. Anchor Books, New York, New York. [1630]

Van Erven, Tim and Peter Harremos (2014), "Rényi divergence and Kullback-Leibler divergence.” IEEE Transactions on Information Theory, 60, 3797-3820. [1652]

Wolfers, Justin and Eric Zitzewitz (2004), “Prediction markets.” Journal of Economic Perspectives, 18, 107-126. [1630]

Co-editor Florian Scheuer handled this manuscript.

Manuscript received 18 September, 2019; final version accepted 25 March, 2020; available online 7 April, 2020. 